\title{
Allheilmittel oder Budgetmimikry: Wie wirksam sind Verschuldungsgrenzen zur Haushaltskonsolidierung?
}

von Uwe Wagschal

Im Zentrum des Beitrags steht die Wirksamkeit von Schulden- und Haushaltsausgleichsregeln. Ausgangspunkt sind dabei die theoretischen Argumente, die für und wider entsprechende Regeln angeführt werden, gefolgt von einer empirischen Untersuchung der Wirksamkeit in unterschiedlichen Kontexten. Hierzu werden die Europäische Union, OECD-Mitglieder, US-amerikanische und deutsche Bundesstaaten sowie Schweizer Kantone betrachtet. Die empirischen Befunde dokumentieren, dass Verschuldungsregeln Finanzkrisen und Höchststände bei der Verschuldung in vielen Ländern nicht verhindern konnten. Allerdings verzeichnen Staaten mit Fiskalregeln im Vergleich durchaus niedrigere Verschuldungszuwächse. Bei der Haushaltskonsolidierung und der Rückführung der Staatsverschuldung bleiben entsprechende Vorschriften hingegen weitgehend wirkungslos. Diskretionäre Politikentscheidungen erweisen sich als bedeutsamer.

This contribution focuses on the efficiency of public debt and balanced budget rules. Based on a number of theoretical approaches, shedding light on the advantages and disadvantages of such rules, the performance of respective policies constitutes the core of the argument. The respective findings are based on a comparative analysis of the European Union, a number of OECD countries, US-American and German states as well as Swiss cantons. Although fiscal rules currently rank high on the public agenda, alternative solutions can conceived. Among the major findings: public debt and balanced budget rules have not been able to avert severe debt crises. However, countries with balanced budget rules perform better than countries without such a rule. Fiscal constraints seem to have almost no impact on the consolidation efforts of indebted countries. In sum, discretionary policies appear more important for public debt reductions than public debt rules.

\section{Einleitung}

In den hoch entwickelten Demokratien dominierte der Anstieg der Staatsverschuldung in den letzten Jahren die politische und ökonomische Agenda. Neben den dramatischen Schuldenkrisen in Griechenland, Italien, Portugal, Spanien und Italien droht inzwischen auch den USA, Japan und verschiedenen osteuropäischen Ländern der Staatsbankrott. Eine zentrale Frage der Politik lautet daher: Wie lässt sich das Anwachsen der Verschuldung wirkungsvoll begrenzen? 
Die am nächsten liegende Antwort auf diese Frage ist ein Verbot zu hoher Staatsverschuldung. Damit scheint man geradezu ein Allheilmittel gegen die Haushaltsmisere gefunden zu haben. Bereits in der Vergangenheit entschieden sich viele Länder für mehr oder weniger strikte Verschuldungsverbote und knüpften die Schuldenaufnahme an bestimmte Regeln oder ökonomische Größen, wie etwa die Investitionsausgaben. Auf der europäischen Ebene wurden im Vertrag von Maastricht 1992 zwei Grenzen für das Haushaltsdefizit (3\% des Bruttoinlandsprodukts (BIP)) und die Gesamtverschuldung (60\% des BIP) eingeführt und zudem ein bail-out anderer Staaten ausgeschlossen. Auch ein Blick in die deutsche Verfassungsgeschichte zeigt, dass solche Regelungen seit dem frühen 19. Jahrhundert existierten und je nach politischer Konjunktur verschärft oder wieder abgeschwächt wurden. ${ }^{1}$ In jüngerer Zeit machte die Schweiz mit der Einführung einer (neuen) Schuldenbremse 2003 auf sich aufmerksam. Die Eidgenossen erzielten selbst in den jüngsten Krisen Budgetüberschüsse. Deutschland implementierte 2009 eine offenere Regel, die sich jedoch stark an der Schweizer Schuldenbremse orientierte.

Doch sind konstitutionelle und gesetzliche Verschuldungsgrenzen wirklich erfolgreich? Nach der Verabschiedung der Maastricht-Regeln stieg die Verschuldung in den Euro-Ländern bis 1999 deutlich schneller als in allen anderen OECD-Staaten. Zunächst bestand somit ein Anreiz, mehr Schulden aufzunehmen. Auch seit der Einführung des Euro 1999 hat sich der Anstieg der Staatsschulden weiter fortgesetzt. Die größte Volkswirtschaft der Welt, die USA, stand im Sommer 2011 sogar kurz vor der Zahlungsunfähigkeit - trotz Schuldengrenze. Erst die im August 2011 in letzter Sekunde erreichte Einigung, die gesetzlich verankerte Schuldenobergrenze um mindestens 2,2 Billionen US-Dollar anzuheben, sicherte dort die Solvenz der öffentlichen Hand. Und das auch nicht zum ersten Mal: Seit 1993 wurde die Schuldenobergrenze der USA in 16 Fällen angehoben, vor der jüngsten Anhebung zuletzt 2010 auf 14,3 Billionen US-Dollar (ca. $88 \%$ des BIP). ${ }^{2}$ Auch in Deutschland verletzte der Bund wiederholt die sogenannte Goldene Regel. Diese bis 2009 im Grundgesetz (Art. 115 a.F. GG) verankerte Budgetregel schrieb vor, dass die Nettokreditaufnahme unterhalb der Investitionsausgaben liegen muss. Zwischen 1992 und 2008 verstieß der Bund

1 Vgl. Blankart, C.B./Fasten, E.R.: Wer soll für die Schulden im Bundesstaat haften? Eine vernachlässigte Frage der Föderalismusreform II, in: Perspektiven der Wirtschaftspolitik 10/1 (2009), 39-59, 42.

2 Austin, A./Levit, M.: The Debt Limit: History and Recent Increases, CRS Report for Congress, Congressional Research Service, Washington D.C., 2010. 
sieben Mal gegen diese Vorschrift und musste die salvatorische Klausel der Abwehr einer Störung des gesamtwirtschaftlichen Gleichgewichts bemühen.

Empirisch sind Verschuldungsregeln in ihrer Wirkung mithin nicht eindeutig, so dass man den politischen Akteuren vor allem aus einer rational-choicePerspektive andere Motive zur Einführung solcher Regelungen unterstellen könnte. Skeptisch formuliert: Es handelt sich bei Verschuldungsgrenzen eher um ein Budgetmimikry. Analog zur Tier- und Pflanzenwelt täuscht die Regierung Bürger, Anleger, Gläubiger und Ratingagenturen, in dem sie den Eindruck einer gesunden Finanzlage erweckt. ${ }^{3}$

Ziel des Beitrages ist daher die Beantwortung der Fragen, ob Schuldenbremsen tatsächlich die Verschuldung dämpfen und ob sie ein wirksames Instrument zur Haushaltskonsolidierung darstellen. Hierzu werden im zweiten Abschnitt zunächst theoretische und empirische Argumente für und gegen diese Hypothese diskutiert. Daran schließt sich eine nähere Beleuchtung der Empirie zur Wirksamkeit entsprechender Regelungen an. Schließlich werden alternative Ansätze diskutiert.

\section{Argumente für und wider Verschuldungsgrenzen}

Die ökonomischen Befürworter von Schuldengrenzen berufen sich im Wesentlichen auf zwei Schulen: die Neue Klassische Makroökonomie und die Konstitutionelle Ökonomik. Die erste Schule formierte sich in den 1970er Jahren explizit gegen den vorherrschenden keynesianischen Steuerungsoptimismus, der sich etwa in dem Mechanismus der Philipps-Kurve manifestiert. Ausgehend von rationalen Erwartungen der Akteure, die sämtliche zur Verfügung stehenden Informationen nutzen, kommen Kydland und Prescott zu dem Ergebnis, dass politische Einflussnahmen entweder wirkungslos sind oder mehr Schaden als Nutzen anrichten. ${ }^{4}$ Regierungen könnten die Fiskalpolitik kaum wirkungsvoll beeinflussen; lediglich über zufällige Störungen könne es zu realen Veränderungen kommen. Kydland und Prescott stellen damit die grundlegende Frage, ob die klassischen Instrumente der Geld- und Fiskalpolitik bei rationalen Erwartungen überhaupt noch wirksam sein können. Hinzu tritt das Problem zeitinkonsistenten Verhaltens von Regierungen, die stets versuchen, mehrere Ziele gleichzeitig zu

3 Der Begriff der Mimikry stammt aus der Biologie und wird für Pflanzen und Tiere verwendet, die sich anderen Arten anpassen, damit sie nicht mehr voneinander unterschieden werden können. Dieses Verhalten dient der Signaltäuschung und soll die Überlebenschancen des Nachahmers vergrößern.

4 Kydland, F. E./Prescott, E. C.: Rules Rather than Discretion. The Inconsistency of Optimal Plans, in: The Journal of Political Economy 85/3 (1977), 473-492. 
erfüllen (wie etwa den Abbau der Arbeitslosigkeit und die Bekämpfung der Inflation). Die aktuelle Schuldenkrise in Europa ist mit ihren massiven Transfers und Rettungspaketen ein Beispiel hierfür. Wurde zum Zeitpunkt der Schaffung der Eurozone die gemeinschaftliche Haftung für Staatsschulden über ein so genanntes bail-out noch kategorisch ausgeschlossen, so wirft man nun selbst geltende Verträge und Bestimmungen (etwa zum Ankauf von Staatsschuldtiteln durch die Europäische Zentralbank) über Bord. Dagegen betonen Kydland und Prescott die Bedeutung einer konsistenten und glaubwürdigen Wirtschaftspolitik mit klaren Regeln. Die Regelbindung der Politik sei einer diskretionären Steuerung bzw. politischen ad hoc-Entscheidungen überlegen, weil nur diese Art der Politik die Entscheidungen privater Wirtschaftssubjekte beeinflussen könne: „[...] policymaker [s] should follow rules rather than discretion“". 5

Am Beispiel der Geldpolitik und der Unabhängigkeit von Notenbanken zeigen die mit dem Nobelpreis für Ökonomie ausgezeichneten Autoren, dass die Inflation nur durch eine strikte und glaubwürdige Regelbindung in Schach gehalten werden kann. Die empirische Evidenz hierfür ist überzeugend und lässt sich an der besseren Inflationsperformanz von Ländern mit unabhängiger Notenbanken ablesen, ${ }^{6}$ die lange Zeit als Vorbild für die Forderung nach einer Ausweitung regelgebundener Politiken galt. Die Einführung der Schuldengrenzen im europäischen Stabilitätspakt stellt ein weiteres Beispiel für regelgebundene Politik dar. Eine - ungeklärte - Frage betrifft jedoch die Umsetzung und Kontrolle solcher Regeln. Das Konzept der Strafzahlungen für Vertragsverletzungen konnte beim Europäischen Stabilitätspakt jedenfalls spätestens seit 2003 nicht mehr glaubhaft durchgehalten werden, als die führenden EU-Länder Deutschland und Frankreich das Defizitverfahren effektiv verhinderten.

Die zweite Schule, die Konstitutionelle Ökonomik, geht davon aus, dass dem Staat eine permanente Wachstumstendenz innewohnt. Der „Leviathan“"7 soll daher durch Verfassungs- und Gesetzesschranken in Zaum gehalten werden. Ähnlich wie Rawls ${ }^{8}$ argumentieren zwei prominente Vertreter dieser Schule, Buchanan und Tullock, ${ }^{9}$ mit einem Schleier der Unwissenheit, der die Bürger in

5 Kydland, F.E./Prescott, E.C., a.a.O., 487.

6 Cukierman, A.: Central Bank Strategy, Credibility, and Independence: Theory and Evidence, London, 1992; Busch, A.: Preisstabilitätspolitik, Opladen, 1995.

7 Buchanan, J.M./Brennan, G.: Besteuerung und Staatsgewalt, Hamburg, 1988.

8 Rawls, J.: A Theory of Justice, Cambridge, 1971.

9 Buchanan, J.M./Tullock, G.: The Calculus of Consent, Ann Arbor, 1962. 
Unkenntnis über ihre sozio-ökonomische Stellung in der Gesellschaft lässt, wodurch für die Wahl fairer und stabiler institutioneller Regelungen gesorgt sei. Schuldengrenzen sind in dieser Lesart Schutzregeln, die nicht zuletzt der Sicherung von Freiheitsrechten dienen.

Neben der Regelbindung und dem Schutz von Freiheitsrechten gibt es weitere Argumente für eine Schuldenbremse. So kann Verschuldung auch als Allmendeproblem interpretiert werden, wonach die politischen Entscheidungsträger möglichst hohe fiskalische Erträge für ihre jeweilige Klientel erzielen möchten. Gelingt dies, so konzentriert sich der Nutzen von Ausgabenprojekten, und damit auch der Staatsverschuldung, auf bestimmte Klientelgruppen, während die Allgemeinheit der Steuerzahler die Kosten dafür zu tragen hat. Ein weiterer Aspekt des common-pool-Problems ist der Haftungsverbund ${ }^{10}$ für die Mitglieder einer Staatengemeinschaft oder eines Föderalstaates. Für Deutschland stellte das Bundesverfassungsgericht diesbezüglich in seinem Urteil zur Haushaltsnotlage der Länder Bremen und Saarland fest: „Befindet sich ein Glied der bundesstaatlichen Gemeinschaft - sei es der Bund, sei es ein Land - in einer extremen Haushaltsnotlage, so erfährt das bundesstaatliche Prinzip seine Konkretisierung in der Pflicht aller anderen Glieder der bundesstaatlichen Gemeinschaft, dem betroffenen Glied [...] Hilfe zu leisten. "11 Solche Hilfszahlungen können sich durch das strategische Verhalten der beteiligten Akteure im so genannten flypaper-Effekt manifestieren, demzufolge Transferzahlungen zu einem Gewöhnungseffekt führen - mit der langfristigen Konsequenz einer höheren Verschuldung. ${ }^{12}$ Eine Schuldenbremse kann insofern einen Beitrag zur Entschärfung des commonpool-Problems leisten.

Ein weiteres Argument für die institutionelle Begrenzung der Staatsverschuldung findet sich im sog. signalling. ${ }^{13}$ Die Existenz von Schuldenregeln bedeutet demnach nichts anderes als ein Signal an den Markt (und die Ratingagenturen), dass der Schuldner es mit seinem Kampf gegen die Verschuldung ernst meint. Regelbindung setzt mithin Signale und erhöht die Glaubwürdigkeit des Schuldners, indem es möglich wird, zwischen (vermeintlich) guten und schlechten Schuldnern zu unterscheiden. Dies führt insgesamt zu mehr Transparenz und stellt für

10 Vgl. Blankart, C.B./Fasten, E.R. a.a.O.

11 BVerfGE 86, 148, 1992.

12 Hines, J. R. Jr./Thaler, R. H.: Anomalies. The Flypaper Effect, in: The Journal of Economic Perspectives 9/4 (1995) 217-226.

13 Cole, H.L./Dow, J./English, W.B.: Default, Settlement, and Signalling: Lending Resumption in a Reputational Model of Sovereign Debt, in: International Economic Review 36/2 (1995), 365-385. 
Gläubiger somit ein wichtiges Datum dar. Der jüngste Streit um die Schuldenobergrenze der USA, in dem die Ratingagentur Moody's den USA mit einer Abwertung ihrer Top-Bonität drohte, falls es zu keiner Einigung zwischen Republikanern und Demokraten kommen sollte, ist ein aktuelles Beispiel für diese Logik. Eine Schuldenregel erweist sich demnach als eine gläubigerfreundliche Einrichtung, was empirisch mit niedrigeren Zinsen (und besseren Ratings) bei Gebietskörperschaften mit Schuldengrenzen belegt werden kann. ${ }^{14}$

Regelgebundene Politik kann jedoch auch negativ auf die Zinskosten und damit in der Tendenz erhöhend auf die Verschuldung wirken, wie Johnson und Kriz in Bezug auf Einnahmeregeln gezeigt haben. ${ }^{15}$ Einer Studie von Poterba und Rueben zufolge ist dieser Effekt aber nur bei Einnahmebegrenzungen zu beobachten. ${ }^{16}$ Ausgaberegeln wirkten demgegenüber dämpfend auf das Zinsniveau, was nach einer Analyse von Lowry und Alt auch auf Regeln zur Schuldenbegrenzung zutrifft. $^{17}$

Diese sowohl theoretischen als auch empirischen Argumente für Verschuldungsgrenzen und Verschuldungsregeln sind jedoch nicht unumstritten. Insbesondere die ökonomische Sichtweise von Kydland und Prescott, wonach diskretionäre Politik einer regelgebundenen Politik systematisch unterlegen ist, kann durch die Krisenerfahrungen der vergangen Jahre in Zweifel gezogen werden. So können starre Regeln die Krise mitunter verschlimmern und im Extremfall sogar die Handlungsfähigkeit des Staates blockieren. Bei der Wahl zwischen Staatsbankrott und unkalkulierbaren Risiken auf der einen und dem Aussetzen von Verschuldungsgrenzen auf der anderen Seite entschieden sich jedenfalls sowohl die USA als auch die Europäische Union für pragmatische Vorgehensweisen und nahmen Abstand von rigiden Schuldenregeln.

Kritik an rigiden Grenzen wird zudem aus demokratietheoretischer Perspektive geübt: Verfassungs- und Gesetzesgrenzen seien vordemokratisch und entzögen dem Parlament sein wichtigstes Recht, die Budgetbewilligung. Zwar hat der Heidelberger Steuerrechtler Mußgnug diesen Vorwurf hauptsächlich gegen die

14 Dornbusch, R.: Fiscal Aspects of Monetary Integration, in: American Economic Review 87/2 (1997), 221-223.

15 Johnson, C./Kriz, K.A.: Fiscal Institutions, Credit Ratings, and Borrowing Costs, in: Public Budgeting \& Finance 25/1 (2005), 84-103.

16 Poterba, J. M./Rueben, K.: State Fiscal Insitutions and the U.S. Municpal Bond Market, in: James M. Poterba, J.M./von Hagen, J.(Hg.): Fiscal Institutions and Fiscal Performance, Chicago, 1999, 181-207.

17 Lowry, R./Alt,K.: A visible hand? Bond markets, political parties, balanced budget laws, and state government debt, mimeo, Harvard University Department of Government, 1997. 
Idee einer Steuerbegrenzung erhoben, doch lässt er sich auch auf Ausgaben- und Verschuldungsgrenzen übertragen. ${ }^{18}$ Hierbei wird freilich übersehen, dass die Gesetze zur Selbstbindung des Ausgabeverhaltens - ähnlich der Gewährung der Unabhängigkeit von Notenbanken - durch demokratisch legitimierte Parlamente verabschiedet wurden. Beschlüsse dieser Art sind daher prinzipiell mit anderen Mehrheiten reversibel.

Schließlich lässt sich auch aus ökonomischer Perspektive Kritik an Verschuldungsgrenzen üben. Zwar stellen diese Limitationen primär Schutzvorrichtungen gegen hohe und ansteigende Staatsschulden dar, doch werden in der Konsequenz nicht selten die durchaus vorhandenen positiven Funktionen der Staatsverschuldung ignoriert. So vermag die Aufnahme von Schuldtiteln etwa Liquiditätsschwankungen im Staatshaushalt zu überbrücken (Überbrückungsfunktion), konjunkturelle Zyklen auszugleichen (Stabilisierungsfunktion) und die finanzielle Last öffentlicher Ausgaben zeitlich zu verteilen (Lastverschiebungsfunktion). Letzteres hatte bereits die deutsche Finanzklassik erkannt: „Ein Staat ohne Staatsschuld tut entweder zu wenig für seine Zukunft, oder er fordert zu viel von seiner Gegenwart“, so Lorenz von Stein im Jahr $1886 .{ }^{19}$ Ferner gibt es ein Nachfrageargument: Anleger und der Kapitalmarkt fordern staatliche Schuldscheine als „,sicheren Hafen“, um über eine risikolose Anlageform zu verfügen. Zu guter Letzt wird in der Literatur auch die Position vertreten, dass Marktkräfte die Verschuldung effizienter begrenzen können als Schuldenbremsen, da Ratingagenturen, die durch ihre Urteile den Preis für Kredite, d.h. die Zinsen, mitbestimmen und so einen durchaus beachtlichen Einfluss auf die staatliche Verschuldungspolitik ausüben könnten. ${ }^{20}$

\section{Der Blick auf die Empirie - zur Performanz von Verschuldungs- regeln}

Wie schneiden nun die Verschuldungsregeln, für die es, wie aufgezeigt, durchaus viele gut begründete Argumente gibt, in der Empirie ab? Hierzu gilt es zunächst zu klären, welche Art von Regeln zur Begrenzung der Staatsverschuldung erkennbar sind bzw. welche allgemeine Systematik von Haushalts- und Budgetregeln identifiziert werden kann, bevor sich der Blick auf die Empirie richtet. Aus-

18 Mußgnug, R.: Verfassungsrechtlicher und gesetzlicher Schutz vor konfiskatorischen Steuern, in: Juristen Zeitung 46/21 (1991), 993-999.

19 Zit. nach Nowotny, E. (Hg.): Öffentliche Verschuldung, Stuttgart, 1979, 3.

20 Ter-Minassian, T.: Fiscal Rules for Subnational Governments: Can they Promote Fiscal Discipline?, in: OECD Journal on Budgeting 6/3 (2007), 108-120. 
gehend von Buchanan lassen sich Verschuldungsregeln generell unter den Begriff der Haushaltsregeln subsumieren. Diese können wiederum in quantitative (bzw. numerische) und prozedurale (bzw. qualitative) Regeln unterschieden werden (vgl. Abb. 1). ${ }^{21}$ Quantitative Schranken sind ergebnisbezogen, d.h. sie geben bestimmte Werte entweder für Steuern, Ausgaben oder Staatsschulden vor, die verbindlich eingehalten werden müssen. Der Extremfall wäre hier ein Verschuldungsverbot, d.h. eine Defizitgrenze, die bei null Prozent des Bruttoinlandsprodukts (BIP) läge. Ausgaben- und Einnahmengrenzen können sich außerdem entweder direkt auf die Gesamtausgaben bzw. Gesamteinnahmen beziehen oder auf einzelne Ausgabenpositionen bzw. einzelne Steuern. Weiter kann man unterscheiden, ob die absolute Höhe festgelegt wird oder nur eine Beschränkung der Zuwachsrate vorliegt. Bei der Verschuldung lassen sich entweder die Defizite direkt begrenzen oder Schuldenobergrenzen angeben. Das bekannteste Beispiel hierfür sind die Konvergenzkriterien des Vertrages von Maastricht, die ein dreiprozentiges Haushaltsdefizit und eine Obergrenze von 60 Prozent der Schulden in Relation zum Bruttoinlandsprodukt zulassen. Die Vorschrift des materiellen Haushaltsausgleichs kann hingegen sowohl als quantitative Barriere als auch als prozedurale Hürde aufgefasst werden, da die Parlamentarier de facto darauf festgelegt werden, einen ausgeglichenen Haushalt zu verabschieden. Ferner können im Abgabenbereich sowohl die Steuersätze als auch die Steuerbemessungsgrundlagen einzelner Steuern begrenzt werden. Letzteres wird eher negativ gesehen, da eine solche Begrenzung ein Einfallstor für Steuerschlupflöcher darstellt. Auch unter der Berücksichtigung ökonomischer Effizienzkriterien und der politischen Durchsetzbarkeit sind explizite Begrenzungen der Bemessungsgrundlagen problematisch. ${ }^{22}$

Prozedurale beziehungsweise qualitative Barrieren lassen sich in spezifische Abstimmungs- und Genehmigungsvorschriften, in direktdemokratische Entscheidungsverfahren sowie in Vorschriften für Gesetze und Programme differenzieren. Bei den spezifischen Abstimmungs- und Genehmigungsvorschriften sind

21 Buchanan, J. M.: Procedural and Quantitative Constitutional Constraints on Fiscal Authority, in Moore, W.S./Penner, R. (Hg.): The Constitution and the Budget, Washington, 1980, 80-84; Reis, K.E.: Fesseln für den Leviathan. Die Steuerrevolte in den Vereinigten Staaten und die Problematik budgetärer Schranken, Pfaffenweiler, 1987.

22 Folkers, C.: Begrenzungen von Steuern und Staatsausgaben in den USA: Eine Untersuchung über Formen, Ursachen und Wirkungen vorgeschlagener und realisierter fiskalischer Restriktionen, BadenBaden, 1993, 54. 
Abbildung 1: Hürden gegen die Staatsverschuldung und andere Formen fiskalpolitischer Begrenzung

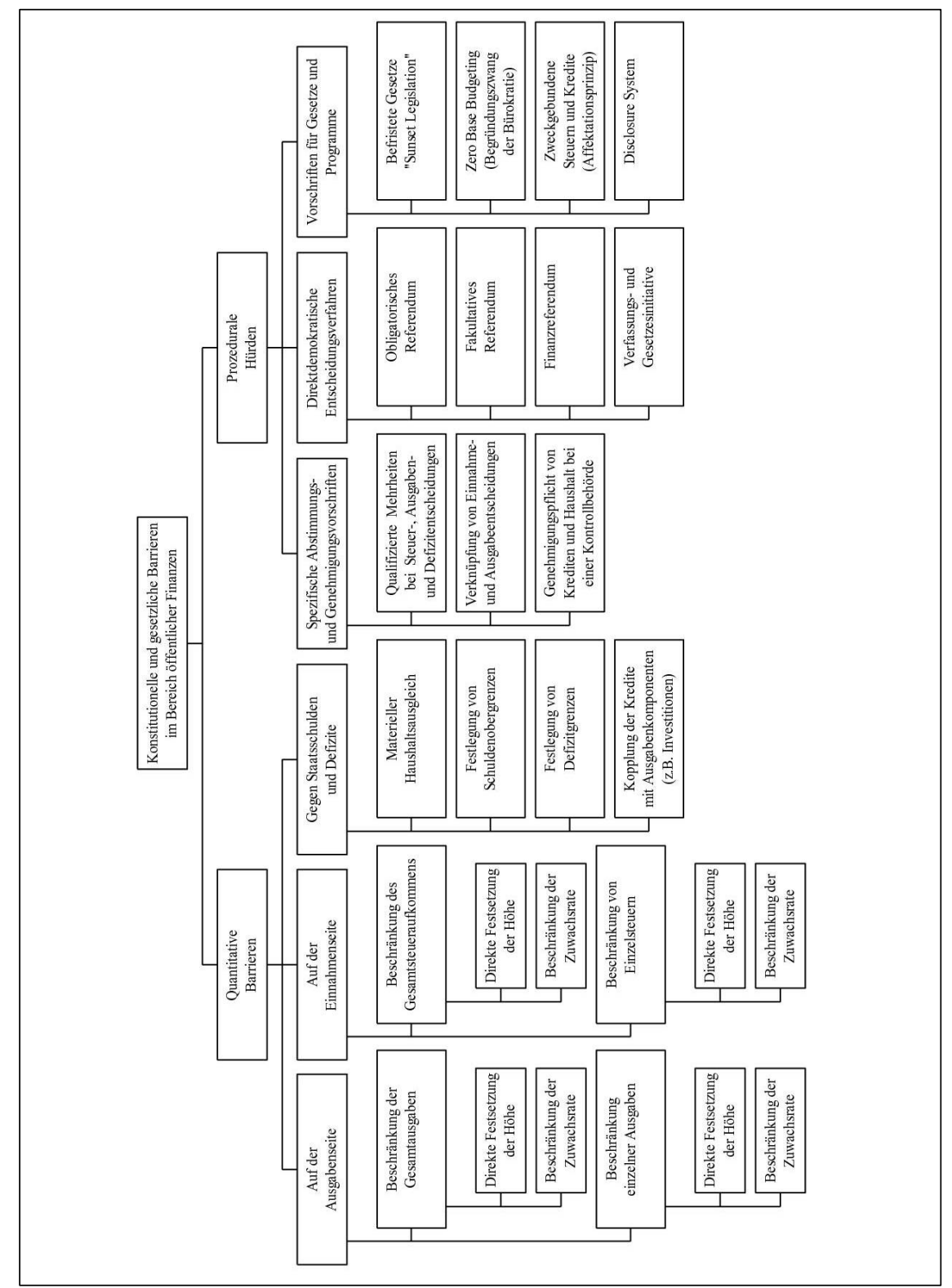

Anmerkung: Systematik auf Basis von Buchanan (1980), Folkers (1983), Reis (1987) sowie eigenen Erweiterungen und Änderungen. 
die qualifizierten Mehrheiten (,supermajorities“') von besonderer Bedeutung, aber auch problematisch, da der Opposition ein Vetorecht erwachsen könnte.

Besonders wirkungsvoll ist die Genehmigungspflicht von Krediten durch eine übergeordnete Kontrollbehörde, so die Erfahrungen auf kommunaler Ebene in Deutschland. ${ }^{23}$ Diese Rechnungskontrolle durch die örtlichen Rechnungsprüfungsämter sowie überörtlich durch die kommunalen Aufsichtsbehörden kann als effizient angesehen werden und erweist sich als wichtige Ursache für die im Vergleich geringe Gemeindeverschuldung in Deutschland. Zudem muss die Kreditaufnahme durch die Aufsichtsbehörden (Landratsämter und Regierungspräsidien) genehmigt werden, die wohl mächtigste Barriere gegen die kommunale Verschuldung. ${ }^{24}$

Auf Basis empirischer Evidenz für die USA und die Schweiz gelten außerdem direktdemokratische Verfahren als wirkungsvolle Bremse. ${ }^{25}$ Dabei muss einerseits zwischen den unterschiedlich ausgeprägten Präferenzen des Elektorats sowie andererseits zwischen den verschiedenen direktdemokratischen Verfahren (top-down versus bottom-up) unterschieden werden. Während etwa der Volksinitiative, auch durch indirekte Wirkungen, ein kostensteigernder Charakter innewohnt, ist das Referendum die stärkste direktdemokratische Expansionshürde.

Eine spezifische Form prozeduraler Grenzen für das Budget sind ferner automatische Kopplungen von Steuereinnahmen an bestimmte vorher festgelegte Ausgaben (,earmarking“). Im bundesdeutschen Haushaltsrecht sind solche Festlegungen wegen des sog. Non-Affektationsprinzips verboten.

$\mathrm{Zu}$ den Vorschriften für Gesetze und Programme sind schließlich die Sunset Legislation, das Zero Base Budgeting sowie das Disclosure-Verfahren zu nennen. Befristete Gesetze mit einem Verfallsdatum (Sunset Legislation) zwingen Exekutive und Legislative dazu, gewünschte Ausgaben- und Einnahmengesetze

23 Banner, G.: Haushaltspolitik und Haushaltskonsolidierung, in: Püttner, G. (Hg.): Handbuch für kommunale Wissenschaft und Praxis, Band 8, Berlin, 1985, 423-440.

24 Zudem dürfen Kredite nur bis zu der Höhe aufgenommen werden, in welcher der gesamte Schuldendienst durch die Einnahmen des Verwaltungshaushaltes geleistet werden kann.

25 Kirchgässner, G./Feld, L. P./Savioz, M. R.: Die direkte Demokratie. Modern, erfolgreich, entwicklungsund exportfähig, München, 1999; Matusaka, J. G.: For the Many or the Few. The Initiative, Public Policy, and American Democracy. Chicago, 2004; Wagschal, U./Obinger, H: Zwischen Reform und Blockade: Plebiszit und der Steuer- und Wohlfahrtsstaat, in: Schmidt, M. G. (Hg.): Wohlfahrtsstaatliche Politik. Institutionen - Prozesse - Leistungsprofil, Opladen, 2000, 92-125. 
nach ihrem Auslaufen neu in das Parlament einzubringen und zu verabschieden. Dies verringert die Pfadabhängigkeit von Entscheidungen. Zero Base Budgeting nimmt das Verhalten der Bürokratie in den Blick. Bei diesem Verfahren müssen für jedes Programm und für jedes Jahr die Kosten und Nutzen ermittelt und mit der Ausgangssituation „ohne Programm“ verglichen werden. Erst bei hinreichend großem Nutzen wird ein Gesetz implementiert. Das Disclosure-System hat zum Ziel, heimliche Steuererhöhungen auszuschalten und für mehr Transparenz zu sorgen. „Kern einer solchen Regel ist, dass das Steueraufkommen nicht ohne expliziten Beschluss der Legislative ansteigen darf. Insofern handelt es sich de facto um ein „Tax Limit“" mit einem besonderen Verfahren, das eingehalten werden muss, wenn dieses überschritten werden soll“". ${ }^{26}$

\section{Die europäischen Verschuldungsgrenzen}

Für die Länder der Euro-Zone wirken die 1992 im Vertrag von Maastricht festgelegten Konvergenzkriterien als verbindliche Verschuldungsgrenzen. Demnach darf das gesamtstaatliche Defizit, wie bereits erwähnt, nicht über 3 Prozent des BIP und der gesamtstaatliche Schuldenstand nicht über 60 Prozent des BIP steigen. Diese Referenzwerte sind im Protokoll 12 des Vertrags über die Arbeitsweise der Europäischen Union (AEUV) ${ }^{27}$ festgeschrieben und bilden den Kern des Stabilitäts- und Wachstumspakts der EU, der die Mitgliedstaaten an diese Haushaltsregeln bindet. Zudem sind die Mitgliedstaaten nach Art 126 AEUV (ehem. Art. 104 EGV) angehalten, übermäßige öffentliche Defizite zu vermeiden. Falls dennoch ein übermäßiges Defizit festgestellt wird, richtet der Europäische Rat eine nicht-öffentliche Empfehlung an den betreffenden Mitgliedstaat, wie dieses abzubauen sei. Die EU-Kommission erstellt daraufhin einen Konvergenzbericht und muss sich dabei an der Entwicklung des Defizits und der Annäherung an den Referenzwert im betreffenden Mitgliedstaat orientieren. Dabei wird unter anderem berücksichtigt, ob der negative Haushaltssaldo die öffentlichen Ausgaben für die Investitionen übertrifft und wie sich Schulden- und Defizitquote jeweils entwickelt haben. Anschließend gibt der Wirtschafts- und Finanzausschuss der EU seine Stellungnahme zu dem Bericht ab. Schließlich entscheidet der Rat mit qualifizierter Mehrheit, ob ein übermäßiges Defizit vorliegt und berücksichtigt dabei sowohl die Empfehlung der EU-Kommission und die Stellungnahme des

26 Reis, K.E., a.a.O., 284.

27 Das benannte Protokoll 12 war ursprünglich dem Vertrag von Maastricht 1992 beigefügt und ist mit dem Vertrag von Lissabon als Anhang in den AEUV übergegangen. 
betroffenen Mitgliedstaats als auch die Gesamtlage. Wird ein übermäßiges Defizit festgestellt, so ergeht an den Mitgliedstaat die Aufforderung, binnen vier Monaten wirksame Maßnahmen dagegen zu ergreifen. Bleibt dies materiell folgenlos, so kann der Ministerrat mit Zweidrittelmehrheit der Euro-Länder Sanktionszahlungen verhängen, die zwischen 0,2 und 0,5 Prozent des BIP betragen können.

So streng diese Regelung zunächst klingen mag, das Verfahren ist bisher nur ein Papiertiger: Deutschland, Frankreich und Portugal verhinderten bereits 2003 den sog. „Blauen Brief“. Im darauffolgenden Jahr konnten Deutschland und Frankreich durch massiven politischen Druck ein Defizitverfahren abwenden, indem eine Sperrminorität gegen den Beschluss organisiert wurde. Bis heute beschloss die EU noch kein einziges Mal Sanktionen gegen Verstöße des Stabilitäts- und Wachstumspaktes, obwohl im Zuge der Finanzkrise seit 2008 gegen 25 der 27 EU-Mitgliedsländer ein Defizitverfahren eingeleitet wurde - lediglich Estland und Schweden konnten ein solches Verfahren vermeiden.

Die Performanz der Euro-Länder in Bezug auf die Einhaltung der Haushaltsregeln des Stabilitätspaktes ist daher auch eher bescheiden (vgl. Abb. 2). Betrachtet man das Drei-Prozent-Defizitkriterium, so wurde es über alle Mitgliedsländer und bezogen auf die jeweilige Mitgliedschaftsdauer in 37,8 Prozent aller möglichen Fälle nicht erfüllt: Relative Spitzenreiter sind Griechenland, Malta und die Slowakei, die das Defizitkriterium bislang in jedem Jahr ihrer EuroMitgliedschaft verletzten. Das Schuldenstandslimit wurde sogar in 51,9 Prozent aller möglichen Fälle durchbrochen. Wiederum liegt Griechenland mit 100 Prozent gemeinsam mit Belgien, Italien, Malta und Österreich an der Spitze. Von den 17 Euroländern haben lediglich Belgien und Spanien die Schuldenstandsquote im Vergleich zum Termin der Euro-Einführung zurückgeführt. Eine kleine Gruppe mit Österreich, Finnland, Italien und den Niederlanden blieb zumindest annähernd stabil.

Dennoch kann für die Zeit vor 1999 ein - nicht intendierter - Effekt dieser Kriterien beobachtet werden. Zwar weisen die durchschnittlichen Haushaltsdefizite nach der Verabschiedung des Vertrags von Maastricht 1992 eine rückläufige Tendenz auf, doch war die Entwicklung deutlich schlechter als bei den übrigen OECD-Ländern. Die Euro-Länder haben also die Zeit vor 1999 zunächst als partiellen Verschuldungsfreibrief genutzt. Nach 1999 ist die Performanz der Euro-Mitgliedsländer bei den zentralen Verschuldungsindikatoren dagegen statistisch nicht mehr signifikant abweichend von den übrigen OECD-Staaten. Außerdem kann ein Konvergenzeffekt identifiziert werden, da die Spannweiten 
Abbildung 2: Verletzungen der Maastricht-Kriterien (1999-2010)

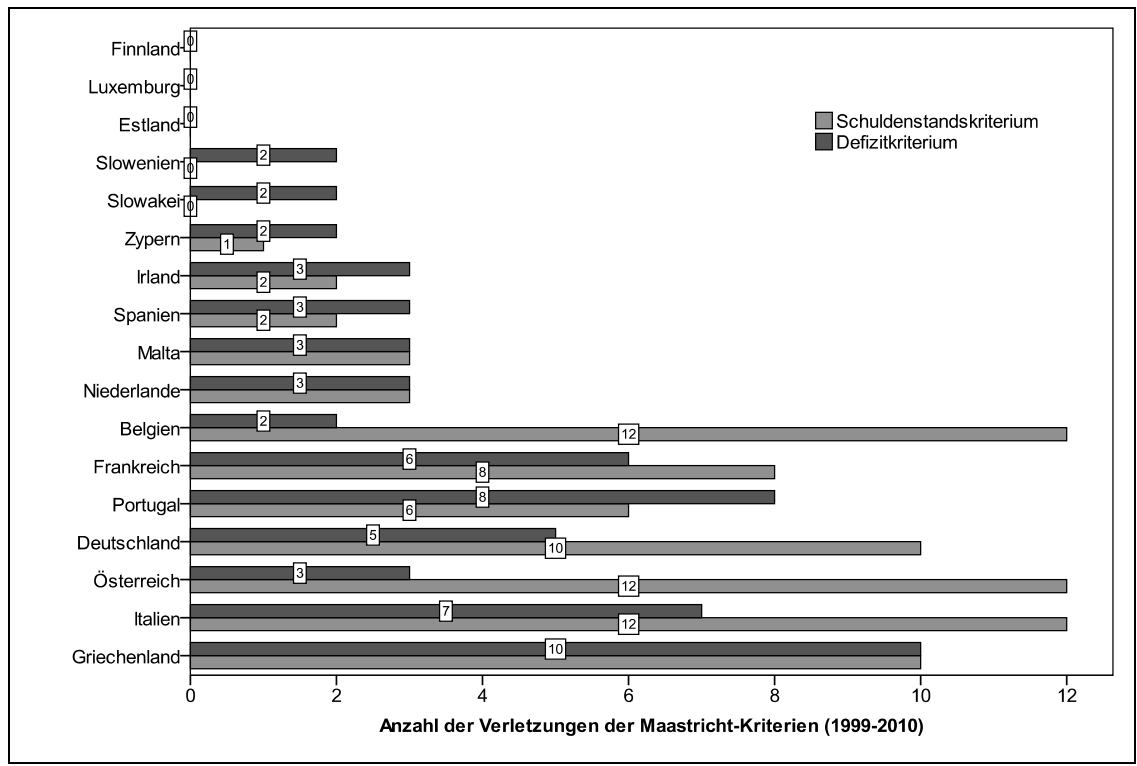

Anmerkungen: Eigene Auswertungen und Berechnungen. Quelle: Eurostat. Indikatoren: „Bruttoschuld des Staates (konsolidiert)“ sowie „Finanzierungssaldo des Staates im Rahmen des Verfahrens bei einem übermässigen Defizit“.

der Haushaltsdefizite zurückgingen und sich - wenn auch mitunter durch buchhalterische Tricks einzelner Staaten - einander annäherten. Insgesamt konnten die europäischen Verschuldungsregeln somit bestenfalls geringe Wirkung entfalten. Das Versagen dieser Regeln kann insofern als eine der Ursachen der gegenwärtigen Schuldenkrise angesehen werden.

Im Zuge der aktuellen Krise reagieren die Mitgliedstaaten der EU und die europäischen Institutionen auf die Ineffizienz des bisherigen Überwachungs- und Sanktionsmechanismus. So verabschiedeten das Europäische Parlament und der EU-Ministerrat im September bzw. Oktober 2011 sechs Reformgesetze (sog. ,Six-Pack") zur Verschärfung des europäischen Stabilitätspakts. Dessen wichtigste Bestandteile sind eine Verschärfung des Verfahrens bei einem übermäßigen Defizit, die Etablierung eines Frühwarnsystems für Haushaltsnotlagen, eine bessere Haushaltsüberwachung, mehr Transparenz im Haushaltsverfahren, institutionelle Änderungen bei der Einleitung eines Defizitverfahrens sowie die Etablierung einer makroökonomischen Koordinierung zum Abbau wirtschaftlicher Ungleichgewichte („Europäische Wirtschaftsregierung“). Künftig kann eine Sanktionsempfehlung der Kommission nur mit einer Zwei-Drittel-Mehrheit im 
Finanzministerrat verhindert werden, wodurch faktisch ein Sanktionsautomatismus bei Regelverstößen implementiert wird. Unklar ist jedoch mit Blick auf den Punkt „makroökonomische Koordinierung“, ob die EU in Zukunft auch Sanktionen gegen Länder mit einem Leistungsbilanzüberschuss verhängen darf - was nicht zuletzt auch Deutschland treffen würde. Die stärkere Fokussierung auf den Schuldenstand, dessen Reduktion nun mit quantifizierenden Vorschriften geregelt ist, kann dagegen als ein Fortschritt im Vergleich zum bisherigen Regelwerk angesehen werden.

Angesichts der bislang fehlenden „ownership“ europäischer Vorschriften, also der mangelnden Akzeptanz bisheriger Verschuldungsregeln in den Nationalstaaten, bleibt allerdings abzuwarten, inwieweit die ab 2012 geltenden verschärften Regeln Prägekraft entfalten.

\section{Verschuldungshürden im internationalen Vergleich}

Sämtliche wichtige internationale Organisationen, die vergleichende Analysen zur Verschuldungspolitik durchführen, verfügen über Indikatoren, die die Existenz und Stringenz von Fiskalregeln erfassen. So entwickelte der Internationale Währungsfonds (IWF) zwei große Datenbanken zur Fiskaltransparenz und betonte schon früh die Wichtigkeit wie die - vermeintliche - Wirksamkeit von Fiskalregeln. ${ }^{28}$ Die OECD veröffentlicht einen Fiscal Rule Index ${ }^{29}$ und argumentiert ebenfalls sehr prononciert für Verschuldungs-, Haushaltsausgleichs- oder Ausgabenregeln. ${ }^{30}$ Und auch die Europäische Kommission konstruierte einen Index zur Erfassung nationaler Haushaltsregeln. ${ }^{31}$ Dieser Fiscal Rule Strength Index (FRSI) misst das institutionelle Framework unterschiedlicher Fiskalregeln

28 International Monetary Fund (IMF): Fiscal Monitor September 2011. Addressing Fiscal Challenges to Reduce Economic Risk, Washington D.C., 2011; vgl. auch IMF: Manual on Fiscal Transparency, Washington D.C., 2007 sowie Kopits, G./Symansky, S.: Fiscal Rules, IMF Occasional Paper 162, Washington D.C., 1998 und Manasse, P.: Deficit Limits, Budget Rules and Fiscal Policy, IMF Working Papers 05/120, Washington D.C., 2005.

29 OECD: Government at a Glance, Paris, 2009, 87.

30 Schick,A.: The Role of Fiscal Rules in Budgeting, in: OECD Journal on Budgeting $3 / 3$ (2003), 7-34; Schick, A.: Post-Crisis Fiscal Rules: Stabilising Public Finance while Responding to Economic Aftershocks, in: OECD Journal on Budgeting 10/2 (2010), 35-51.

31 European Commission: Public Finances in EMU 2009, Directorate-General for Economic and Financial Affairs, European Economy 5/2009, 2009, 87 ff.; Deroose, S./Moulin, L./Wierts, P.: National expenditure rules and expenditure outcomes. Empirical evidence for EU Member States, in: Wirtschaftspolitische Blätter 1/2006, 27-42. 
in einzelnen Ländern, wobei für fünf unterschiedliche Bereiche Punkte vergeben werden. Diese Bereiche sind:

- Gesetzliche bzw. konstitutionelle Verankerung der Regeln,

- Anpassungsflexibilität (negativ) bzw. Rigidität der Regeln,

- Überwachung und Regelbefolgung,

- Sanktionsmechanismen und

- $\quad$ offentliche Sichtbarkeit der Regeln.

Die Daten werden sowohl für die unterschiedlichen staatlichen Ebenen erhoben als auch für verschiedene Arten: Budgetausgleichs-, Schulden-, Ausgaben- sowie Einnahmeregeln (vgl. Tab. 1). Dabei ist die Variation beachtlich, wobei Haushaltsausgleichsregeln dominieren, gefolgt von Schulden- und Ausgabenregeln. Besonders bemerkenswert ist jedoch die Tatsache, dass die Zahl der nationalen Normierungen seit 1990 stark angestiegen ist - parallel zum Anstieg der Verschuldungsquoten. Somit stellt sich für die Interpretation ein erhebliches Kausalitätsproblem: Folgt die Implementation einer wachsenden Verschuldung oder sind die Regeln unwirksam und dienen - im Sinne des Budgetmimikry - nur zur Beruhigung von Wählern, Anlegern und Banken?

Außerdem sind einige Regeln für den Schuldenabbau auch nicht notwendigerweise positiv: So können Einnahmeregeln eine Konsolidierung sogar behindern, wenn damit Steuererhöhungen verhindert werden. Sie tragen nur dann wirksam zur Konsolidierung bei, wenn sie mit einem Automatismus zur Einnahmeerhöhung bei einem hohen Defizit verbunden sind - wie es zum Beispiel in manchen Schweizer Kantonen der Fall ist.

Wirft man den Blick auf das Haushaltsdefizit, zeigt sich in der empirischen Überprüfung dagegen, dass im Durchschnitt des Zeitraumes von 2001 bis 2009 eine klar dämpfende Wirkung auf die durchschnittliche Höhe des Haushaltsdefizits erkennbar wird. Dieser Befund wird auch durch multivariate Analysen der Europäischen Kommission gestützt: Je stärker der Fiscal Rules Index für ein Land ausfällt, desto stärker wird dort das (konjunkturbereinigte) Primärdefizit gedämpft (vgl. Abb. 3). ${ }^{32}$ 
Tabelle 1: Zahl der EU-Mitgliedstaaten mit unterschiedlichen Ausprägungen von Haushaltsregeln (2008)

\begin{tabular}{|c|c|c|c|c|c|c|}
\hline \multirow[t]{2}{*}{$\begin{array}{l}\text { Haushalts- } \\
\text { ausgleichs- } \\
\text { regeln }\end{array}$} & $\begin{array}{l}\text { "Goldene } \\
\text { Regeln" }\end{array}$ & $\begin{array}{l}\text { Regeln für } \\
\text { ausgeglich- } \\
\text { ene Budgets }\end{array}$ & $\begin{array}{c}\text { Nominale } \\
\text { Obergrenze }\end{array}$ & \begin{tabular}{|c|} 
Obergrenze \\
in $\%$ des \\
BIP \\
\end{tabular} & $\begin{array}{c}\text { Strukturelle } \\
\text { Regeln }\end{array}$ & Gesamt \\
\hline & 5 & 10 & 7 & 1 & 3 & 26 \\
\hline \multirow[t]{2}{*}{$\begin{array}{l}\text { Schulden- } \\
\text { regeln }\end{array}$} & $\begin{array}{c}\text { Nominale } \\
\text { Schulden- } \\
\text { obergrenze }\end{array}$ & $\begin{array}{c}\text { Schulden- } \\
\text { obergrenze } \\
\text { in } \% \text { des } \\
\text { BIP }\end{array}$ & \begin{tabular}{|c|} 
Schulden- \\
obergrenze \\
in Relation \\
zur Rück- \\
zahlungs- \\
kapazität \\
\end{tabular} & \multicolumn{2}{|c|}{$\begin{array}{l}\text { Andere } \\
\text { Regeln }\end{array}$} & Gesamt \\
\hline & 5 & 3 & 8 & \multicolumn{2}{|c|}{2} & 18 \\
\hline \multirow[t]{2}{*}{$\begin{array}{l}\text { Ausgaben- } \\
\text { regeln }\end{array}$} & $\begin{array}{l}\text { Nominale } \\
\text { Ausgaben- } \\
\text { obergrenze }\end{array}$ & $\begin{array}{c}\text { Reale } \\
\text { Ausgaben- } \\
\text { obergrenze }\end{array}$ & $\begin{array}{c}\text { Nominale } \\
\text { Ausgaben- } \\
\text { wachstums- } \\
\text { rate }\end{array}$ & \begin{tabular}{|c|} 
Reale \\
Ausgaben \\
wachstums- \\
rate \\
\end{tabular} & $\begin{array}{l}\text { Andere } \\
\text { Regeln }\end{array}$ & Gesamt \\
\hline & 5 & 2 & 4 & 3 & 3 & 17 \\
\hline \multirow[t]{2}{*}{$\begin{array}{l}\text { Einnahme- } \\
\text { regeln }\end{array}$} & $\begin{array}{c}\text { Steuerlast } \\
\text { in } \% \text { des } \\
\text { BIP }\end{array}$ & $\begin{array}{c}\text { Regeln in } \\
\text { Zusammen- } \\
\text { hang mit } \\
\text { Steuersät- } \\
\text { zen }\end{array}$ & $\begin{array}{c}\text { Zuteilung } \\
\text { von zusätz- } \\
\text { lichen } \\
\text { Einnahmen }\end{array}$ & \multicolumn{2}{|c|}{$\begin{array}{l}\text { Andere } \\
\text { Regeln }\end{array}$} & Gesamt \\
\hline & 0 & 1 & 4 & \multicolumn{2}{|c|}{1} & 6 \\
\hline
\end{tabular}

Quelle: European Commission, a.a.O., 90.

Allerdings ist eine Dämpfung des Anwachsens nicht gleichbedeutend mit einem Abbau der Staatsverschuldung. Hauskonsolidierung ist ein mehrjähriger Prozess der Rückführung von Schuldenstand und Defizit. Zur Beurteilung der Konsolidierungsanstrengungen in den OECD-Ländern (aber auch in den deutschen Bundesländern) hat der Autor daher einen Konsolidierungsindikator entwickelt der von 1 bis 10 (= stärkste Konsolidierungsanstrengung) skaliert ist. ${ }^{33}$ Auf der Basis von vier Kriterien werden damit die Konsolidierungsanstrengungen der Untersuchungsländer ermittelt: (1) Eine Untersuchungsperiode von jeweils zwei Jahren, (2) das Niveau des Primärsaldos (in Prozent des BIP), gemessen am Durchschnitt des betrachteten Zeitraums, (3) die Entwicklung der Staatsverschuldung (in Prozent des BIP = Staatsschuldenquote) während der Untersuchungsperiode

33 Wagschal, U./Wenzelburger, G./Metz, T./Jäkel, T.: Konsolidierungsstrategien der Bundesländer, Gütersloh, 2009; Wagschal, U./Wenzelburger, G.: Determinanten der Haushaltskonsolidierung der Bundesländer (1992-2006), in: Zeitschrift für Vergleichende Politikwissenschaft, 3/1 (2009) 33-58. 
Abbildung 3: Fiskalregeln und Haushaltsdefizit (2001-2009)

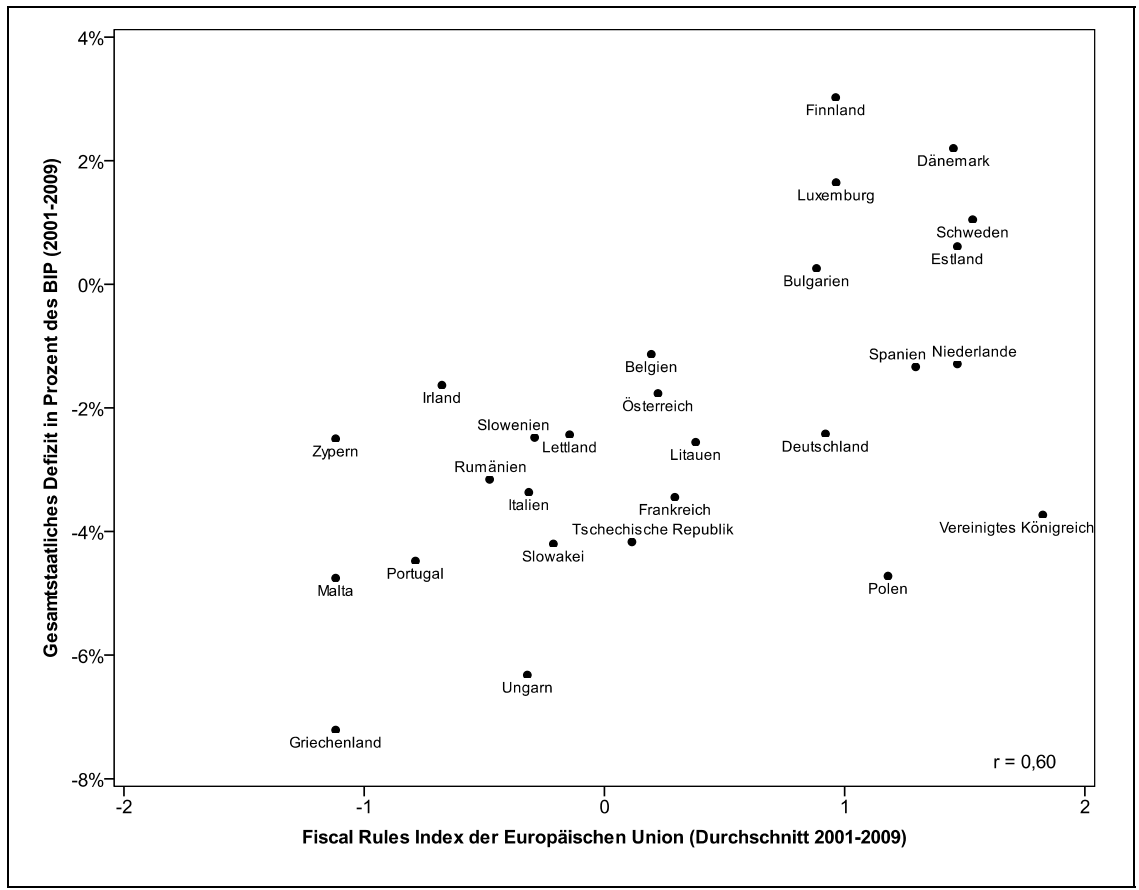

Anmerkung: Daten aus European Commission, Fiscal Rule Strength Index, a.a.O., sowie Eurostat; eigene Berechnungen.

(Differenz im letzten Jahr vor der Konsolidierung im Vergleich zum letzten Jahr der Untersuchungsperiode) und (4) die Entwicklung der Staatsverschuldung (in Prozent des BIP) in den drei Perioden nach Ablauf des Untersuchungszeitraums („Erfolgskriterium“). Zur Darstellung wurde über alle (gleitenden) Untersuchungsperioden das arithmetische Mittel gebildet.

Die Auswertung in Abb. 4 zeigt keinen statistischen Zusammenhang zwischen den Konsolidierungsanstrengungen und dem Fiskalregelindex der EU. Gleiches gilt für ein multivariates Untersuchungsdesign, welches für andere Erklärungsfaktoren zusätzlich kontrolliert. Der Befund des internationalen Vergleichs ist mithin eindeutig: Für Konsolidierungen sind Verschuldungs- bzw. Fiskalregeln nicht wirksam, diese folgen anderen Logiken. Für den Abbau der Staatsverschuldung braucht es also andere Instrumente und Strategien als die bloße Fixierung auf Verschuldungsregeln. 
Abbildung 4: Fiskalregeln und Konsolidierungsanstrengungen (2001-2009)

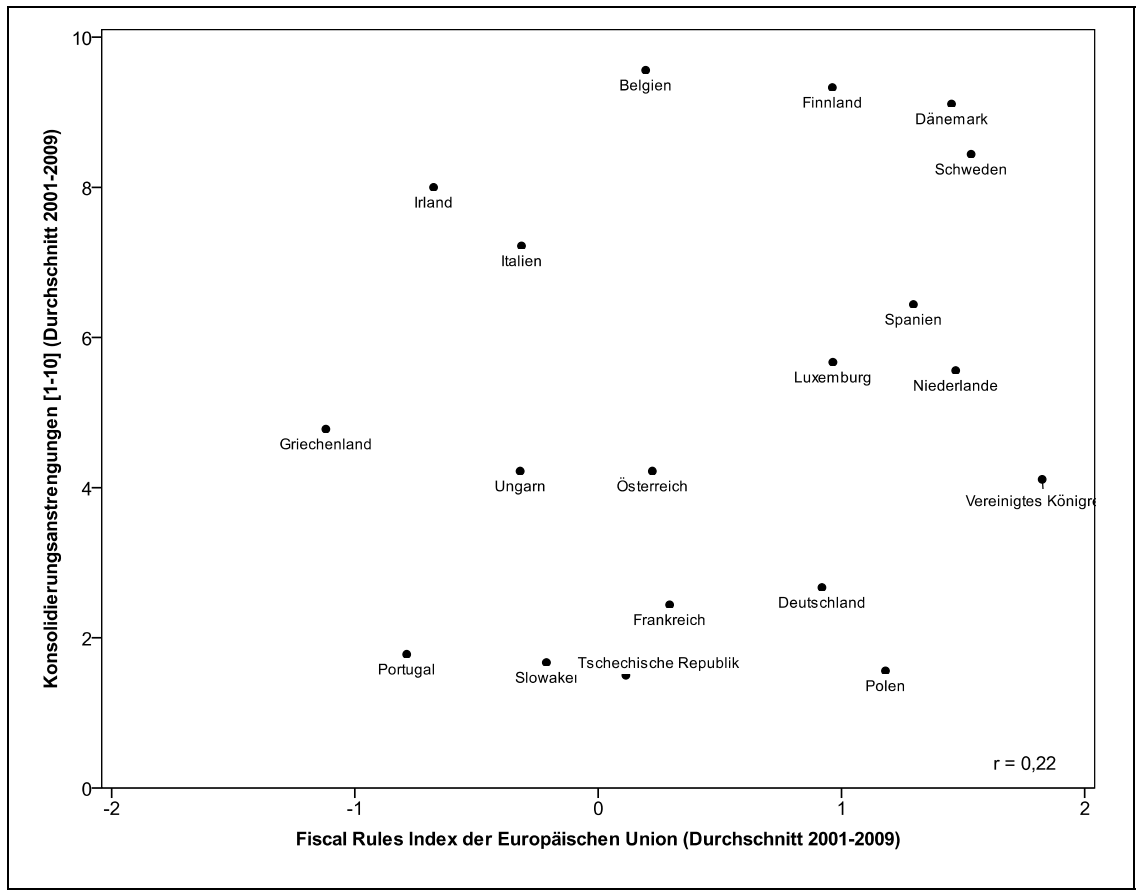

Anmerkung: Daten aus European Commission, Fiscal Rule Strength Index, a.a.O., sowie eigene Berechnungen anhand von $O E C D$-Daten.

\section{Verfassungshürden im Vergleich der US-Bundesstaaten}

In den Vereinigten Staaten haben Budgetregeln eine lange Tradition: Die ersten dieser so genannten ,tax and expenditure limitations“ (TEL) wurden bereits im 19. Jahrhundert im Gefolge einer Bankenkrise als „balanced budget amendments" in einzelne Verfassungen der amerikanischen Bundesstaaten aufgenommen. ${ }^{34}$ Rhode Island machte hier 1842 den Anfang, bis 1900 verankerten 37 Bundesstaaten entsprechende Regeln in ihrer Verfassung. Besonders in den Fokus geriet die Steuerrevolte der späten 1970er Jahre mit der „Proposition 13“ in Kalifornien, in deren Nachgang weitere Steuer- und Ausgabenbegrenzungen in die einzelstaatlichen Verfassungen aufgenommen wurden. Bis Anfang der 1980er Jahre implementierten insgesamt 27 Bundesstaaten weitere verschärfende

34 Penner, R.G./Weisner, M.: Do State Budget Rules Affect Welfare Spending?, The Urban Institute, Occasional Paper Nr. 43, Washington D.C., 2001. 
Begrenzungsvorschriften. Diese Regeln waren aber nicht ohne Risiko, wie die Fast-Pleite von Kalifornien dokumentiert. Trotz einer der schärfsten Haushaltsausgleichsregeln im Vergleich der Bundesstaaten untereinander ließ sich eine Verschlechterung der Haushaltslage dort nicht verhindern, was unter anderem auch auf fehlende Möglichkeiten zur Einnahmeerzielung aufgrund ebendieser Regeln zurückzuführen ist. ${ }^{35}$

Im Jahr 2008 hatten mit Ausnahme Vermonts alle Bundesstaaten der USA zumindest eine Defizitbegrenzungsregel implementiert. Folgende Haushaltsausgleichsvorschriften sind in den Verfassungen bzw. in Gesetzen verankert: ${ }^{36}$ (1) Der Gouverneur muss ein ausgeglichenes Budget vorlegen (in 44 von 50 Bundesstaaten; Stand: 2008), (2) die Parlamente müssen ein ausgeglichenes Budget verabschieden (in 41 von 50 Bundesstaaten) und (3) Defizite können nicht übertragen werden (in 38 von 50 Bundesstaaten). Unterschiedliche Vergleichsstudien zeigen ein Anwachsen der Haushaltsausgleichsregeln im Zeitverlauf. ${ }^{37}$ Darüber hinaus sind in den meisten Bundesstaaten auch Formen von Steuer- und Ausgabenbegrenzung zu finden. Allerdings ergibt sich hier bei näherer Analyse eine große Variation sowohl in der Ausgestaltung dieser Begrenzungsregeln als auch, und vor allem, in deren Reichweite und Wirksamkeit. Auch die Sanktionsmöglichkeiten bei einer Regelverletzung differieren stark. So kann etwa der Finanzminister in Alabama bei einer Verletzung zu 5000 Dollar Strafe und zu einer Gefängnisstrafe von bis zu zwei Jahren verurteilt werden. ${ }^{38}$

Eine weitere Hürde gegen die Staatsverschuldung sind spezifische Abstimmungs- und Genehmigungsvorschriften. Ein Beispiel hierfür ist die so genannte „supermajority“, wonach eine qualifizierte Mehrheit zur Aufnahme von Schulden erforderlich ist und eine absolute oder gar eine relative Mehrheit im Parlament nicht ausreicht. Die Opposition hat in diesem Fall bei Defizitentscheidungen fast immer ein Vetorecht, womit die Aufnahme von Schulden unwahrscheinlicher wird. Entsprechende Mehrheitserfordernisse haben sich

35 Gamage, D.: Coping through California's Budget Crises in Light of Proposition 13 and California's Fiscal Constitution, in: Citrin, J./Martin, I. (eds.): After the Tax Revolt. California's proposition 13 turns 30, Berkeley, 2009.

36 National Conference of State Legislatures: NCSL Fiscal Brief: State Balanced Budget Provisions, Washington D.C., 2010.

37 Hou Y./Smith, D.L.: A Framework for Understanding State Balanced Budget Requirement Systems: Reexamining Distinctive Features and an Operational Definition, in: Public Budgeting and Finance 26/3 (2006), 22-45.

38 National Conference of State Legislatures: a.a.O., 9. 
während der vergangenen beiden Dekaden in den Vereinigten Staaten zunehmend durchgesetzt.

Die empirische Evidenz zur Wirksamkeit der Schuldengrenzen und Haushaltsausgleichsregeln in den USA ist uneinheitlich. ${ }^{39}$ Bereits die erste Vergleichsstudie von Bohn und Inman erbrachte ambivalente Ergebnisse. ${ }^{40}$ Insbesondere Poterba verweist jedoch immer wieder auf die positiven Wirkungen. ${ }^{41}$ Dem halten Penner und Weisner negierend entgegen: „Whether or not a state has strict budget rules seems to be largely unimportant to the way in which its welfare spending responds to the ups and downs of the business cycle. “42 $\mathrm{Zu}$ einem ähnlichen Ergebnis kommt auch Levinson, der bei Bundesstaaten mit stärkeren Budgetregeln auch größere Konjunkturausschläge sieht. ${ }^{43}$ Insgesamt zeigen die Ergebnisse des US-Bundesstaatenvergleichs, dass die Art der Regel und die Stringenz der Regelbefolgung als zentrale Variablen anzusehen sind.

\section{Verschuldungsgrenzen in Deutschland}

In Deutschland reichen Regeln zur Schuldenbegrenzung ebenso wie in den USA historisch weit zurück; seit 1820 sind sie in Deutschlands Verfassungen nachzuweisen. $^{44}$ Auch institutionell wurden entsprechende Vorschriften mit der Gründung der preußischen Hauptverwaltung der Staatsschulden im selben Jahr verankert. Auf nationaler Ebene waren die Regelungen der Paulskirchenverfassung von 1849 eher weich und hätten der Reichsregierung nach $\S 51$ Verfassung des Deutschen Reiches vom 28. März 1849 viel Spielraum für eine extensive Verschuldung eingeräumt:

"Die Reichsgewalt ist befugt, in außerordentlichen Fällen Reichssteuern aufzulegen und zu erheben oder erheben zu lassen, sowie Anleihen zu machen oder sonstige Schulden zu contrahiren."

39 Vgl. z.B. Inman, R.: Do Balanced Budget Rules Work? U.S. Experience and Possible Lessons for the EMU, NBER Working Paper 5838, 1998; Penner, R.G./Weisner, M.: a.a.O.; Poterba, J.M./Rueben, K.S., a.a.O.

40 Bohn, H./Inman, R.P.: Balanced-budget Rules and Public Deficits: Evidence from the U.S. States, Carnegie-Rochester Conference Series on Public Policy 45 (1996), 13-76.

41 Poterba, J.M.: Do Budget Rules Work? NBER Working Paper 5550, 1997.

42 Penner, R.G./Weisner, M.: a.a.O., 16.

43 Levinson, A.: Budget Rules and State Business Cycles: A Comment, in: Public Finance Review 35/4 (2007) 545-549.

44 Blankart, C.B./Fasten, E.R., a.a.O. 
Auch die föderalistisch orientierte Reichsverfassung von 1871 war - wie der textidentische Artikel der Verfassung des Norddeutschen Bundes - in ihren Bestimmungen zur Staatsverschuldung nicht strenger. So hieß es in Art. 73 der Reichsverfassung vom 1. Januar 1871:

"In Fällen eines außerordentlichen Bedürfnisses können im Wege der Bundesgesetzgebung die Aufnahme einer Anleihe, sowie die Übernahme einer Garantie zu Lasten des Bundes erfolgen.“

Eine Möglichkeit, exzessive Verschuldung in den Griff zu bekommen, sind automatische Steuererhöhungen. Solche Regelungen existieren heute in mehreren Schweizer Kantonen (z.B. in St. Gallen), ihr Grundgedanke ist historisch aber auch in Deutschland nicht unbekannt. In der Verfassung des Deutschen Reichs von 1871 findet sich in Artikel 70 eine ähnliche Vorschrift zum Verschuldungsausgleich, wodurch die schwache Regelung aus Art. 73 indirekt deutlich stärker wirkt. Dort werden die einzelnen Bundesstaaten gezwungen, ihre Matrikularbeiträge an das Reich zu erhöhen, wenn dieses seine Aufgaben nicht mehr erfüllen kann:

"Zur Bestreitung aller gemeinschaftlichen Ausgaben dienen zunächst die etwaigen Überschüsse der Vorjahre, sowie die aus den Zöllen, den gemeinschaftlichen Verbrauchssteuern und aus dem Post- und Telegraphenwesen fließenden gemeinschaftlichen Einnahmen. Insoweit dieselben durch diese Einnahmen nicht gedeckt werden, sind sie, so lange Reichssteuern nicht eingeführt sind, durch Beiträge der einzelnen Bundesstaaten nach Maßgabe ihrer Bevölkerung aufzubringen, welche bis zur Höhe des budgetmäßigen Betrages durch den Reichskanzler ausgeschrieben werden.“

Auch die Aussagen zur Schuldenreglementierung in der Weimarer Reichsverfassung waren noch sehr schwach und sicherlich kein Hindernis für die enorme Reichsverschuldung der nachfolgenden Jahre. Art. 87 WRV vom 11. August 1919 lautet:

„Im Wege des Kredits dürfen Geldmittel nur bei außerordentlichem Bedarf und in der Regel nur für Ausgaben zu werbenden Zwecken beschafft werden. Eine solche Beschaffung sowie die Übernahme einer Sicherheitsleistung zu Lasten des Reichs dürfen nur auf Grund eines Reichsgesetzes erfolgen.“

Fast textidentisch zur Weimarer Reichsverfassung stellten sich die Regelungen in Art. 115 Grundgesetz vom 23.05.1949 dar, die wie die Weimarer Reichsverfassung eine Orientierung an den Investitionen vorsahen, wobei Investitionen als „werbender Zweck“ benannt wurden:

„Im Wege des Kredites dürfen Geldmittel nur bei außerordentlichem Bedarf und in der Regel nur für Ausgaben zu werbenden Zwecken und nur auf Grund eines Bun- 
desgesetzes beschafft werden. Kreditgewährungen und Sicherheitsleistungen zu Lasten des Bundes, deren Wirkung über ein Rechnungsjahr hinausgeht, dürfen nur auf Grund eines Bundesgesetzes erfolgen. In dem Gesetze muß die Höhe des Kredites oder der Umfang der Verpflichtung, für die der Bund die Haftung übernimmt, bestimmt sein."

Trotz nahezu identischer Rechtsvorschrift gab es bemerkenswerte Unterschiede bei der Verschuldungsperformanz zwischen den Anfangsjahren der Bundesrepublik und der Weimarer Republik. Letztere litt unter einer massiven Verschuldung, die im Wesentlichen durch eine abhängige Notenbank und die damit verbundene Inflation befeuert wurde. Hier wird deutlich, welche herausragende Rolle Kontextfaktoren, wie etwa Reparationszahlungen, anhaltend hohe Arbeitslosigkeit und politische Instabilität, spielen. Die obige Investitionsregel wurde später mit Art. 115 Abs. 1 GG in der Fassung von 1969 um eine abschwächende salvatorische Klausel ergänzt, wonach die Kreditaufnahme die Investitionen nur dann überschreiten darf, wenn dies zur konjunkturellen Stützung notwendig ist:

„Die Aufnahme von Krediten sowie die Übernahme von Bürgschaften, Garantien oder sonstigen Gewährleistungen, die zu Ausgaben in künftigen Rechnungsjahren führen können, bedürfen einer der Höhe nach bestimmten oder bestimmbaren Ermächtigung durch Bundesgesetz. Die Einnahmen aus Krediten dürfen die Summe der im Haushaltsplan veranschlagten Ausgaben für Investitionen nicht überschreiten; Ausnahmen sind nur zulässig zur Abwehr einer Störung des gesamtwirtschaftlichen Gleichgewichts. Das Nähere wird durch Bundesgesetz geregelt.“

Diese von 1969 bis 2009 im Grundgesetz verankerte, so genannte „Goldene Regel “ war rein ökonomisch begründet und koppelte eine im Prinzip objektbezogene Verschuldung an Ertrag bringende Investitionen. Problematisch waren dabei die Unbestimmtheit des Investitionsbegriffs sowie fortbestehende Abgrenzungsprobleme und die Möglichkeit des Zusammenspiels mit Art. 109 GG, der die Beachtung des gesamtwirtschaftlichen Gleichgewichts vorsah und als Hebel zur Umgehung der „Goldenen Regel“ diente. ${ }^{45}$ Zudem ist mit Blick auf die Investitionen, die wegen ihrer unterstellten Wachstumswirkung zumeist positiv gesehen werden, kritisch anzumerken, dass Bundesländer wie Bremen, die auf eine explizite Wachstumsstrategie zur Entschuldung gesetzt haben, damit ge-

45 Sachverständigenrat zur Begutachtung der gesamtwirtschaftlichen Entwicklung (SVR): Staatsverschuldung wirksam begrenzen, Expertise im Auftrag des Bundesministers für Wirtschaft und Technologie, Wiesbaden, 2007. 
scheitert sind. ${ }^{46}$ Vergleicht man schließlich anhand der bis 2009 gültigen Verschuldungsgrenze des Art. 115 GG die Nettokreditaufnahme mit den eigenfinanzierten Investitionsausgaben im Bund und in den Ländern, dann wird im Zeitraum von 1992 bis 2006 in 53 Landeshaushalten eine Neuverschuldung über der Höhe der eigenfinanzierten Ausgaben erkennbar (vgl. Abb. 5). Damit verletzten die Haushalte rein rechnerisch die so genannte „Goldene Regel“, die analog zu

Abbildung 5: Anzahl der Jahre, in denen die Nettokreditaufnahme im Haushaltsvollzug größer war als die eigenfinanzierten Investitionsausgaben (1992-2006)

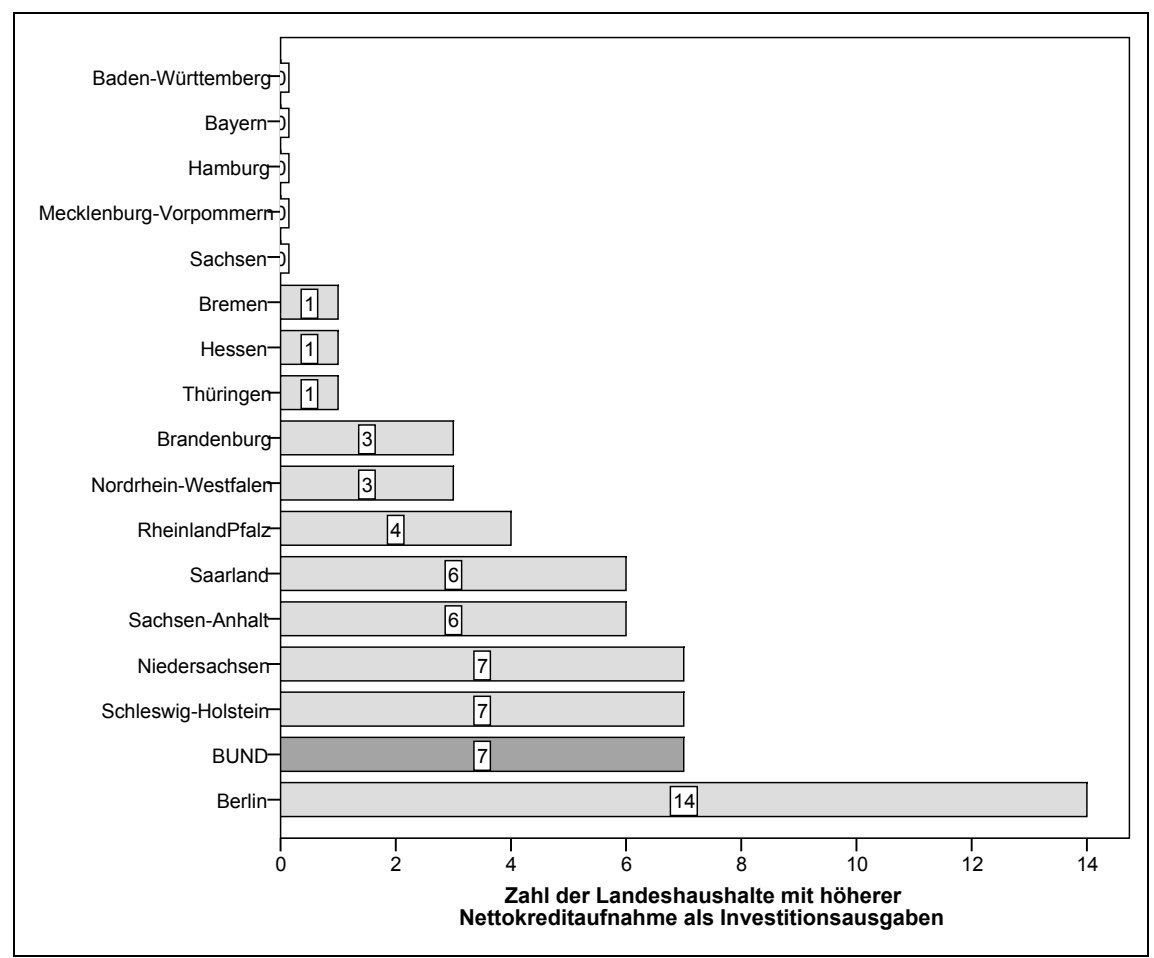

Anmerkung: Datenquelle sind die Kassenergebnisse des öffentlichen Gesamthaushaltes des Statistischen Bundesamtes (Fachserie 14, Reihe 2), Jahrgänge 1992-2006. Die Bruttoinvestitionen ergeben sich aus der Summe der Hauptgruppen 7 und 8 des Gruppierungsplans der Haushaltssystematik von Bund und Ländern. Abzüglich der erhaltenen Investitionszuweisungen (Obergruppen 33 und 34) ergeben sich die eigenfinanzierten Investitionen. Dieser Größe wird die Nettokreditaufnahme gegenübergestellt: Diese ergibt sich aus der Schuldenaufnahme am Kreditmarkt (Obergruppe 32) abzüglich der Tilgungsausgaben am Kreditmarkt (Obergruppe 59).

46 Hickel, R./Lang, W.: Sanieren und Investieren. Eine kritische Bilanz zu den ökonomischen und fiskalischen Effekten der bisherigen Sanierungspolitik des Landes Bremen, Bremen, 2003 
Art. $115 \mathrm{GG}$ auch in den meisten Landesverfassungen enthalten ist. ${ }^{47}$ Selbst bei ausschließlicher Betrachtung der Bruttoinvestitionen übersteigt die Nettoneuverschuldung im Haushaltsvollzug in immerhin noch 22 Fällen die Investitionsausgaben.

Die Grundgesetzänderung der zweiten Föderalismusreform von 2009 zielte folglich darauf ab, diese Regel zu verschärfen, da der bisherige Investitionsbegriff zu ungenau schien. Mit dem Haushaltsjahr 2011 gilt nun die neue ,Schuldenbremse“ des Art. 115 GG in der Fassung vom 29.07.2009, deren Vorbild das Schweizer Pendant von 2003 ist:

„(1) Die Aufnahme von Krediten sowie die Übernahme von Bürgschaften, Garantien oder sonstigen Gewährleistungen, die zu Ausgaben in künftigen Rechnungsjahren führen können, bedürfen einer der Höhe nach bestimmten oder bestimmbaren Ermächtigung durch Bundesgesetz.

(2) Einnahmen und Ausgaben sind grundsätzlich ohne Einnahmen aus Krediten auszugleichen. Diesem Grundsatz ist entsprochen, wenn die Einnahmen aus Krediten 0,35 vom Hundert im Verhältnis zum nominalen Bruttoinlandsprodukt nicht überschreiten. Zusätzlich sind bei einer von der Normallage abweichenden konjunkturellen Entwicklung die Auswirkungen auf den Haushalt im Auf- und Abschwung symmetrisch zu berücksichtigen. Abweichungen der tatsächlichen Kreditaufnahme von der nach den Sätzen 1 bis 3 zulässigen Kreditobergrenze werden auf einem Kontroll-

47 Die Regelgrenze von Art. 115 GG a.F. bezieht sich nur auf die Haushaltsaufstellung bzw. den Haushaltsplan. Ein weiteres Problem ist die weite haushaltsrechtliche Definition des Investitionsbegriffs (vgl. SVR: Widerstreitende Interesen - ungenutzte Chancen, Jahresgutachten 2006/07, 308). Haushaltsgrundsätzegesetz ( 110 II 2 HGrG) und Bundeshaushaltsordnung ( 13 III 2 BHO) definieren Investitionen als Ausgaben für Baumaßnahmen, den Erwerb von beweglichen und unbeweglichen Sachen, den Erwerb von Beteiligungen und sonstigem Kapitalvermögen, Darlehen, die Inanspruchnahme aus Gewährsleistungen sowie Zuweisungen und Zuschüsse für Ausgaben zum Zwecke der genannten Punkte. Im Investitionsbegriff des Art. 115 GG a.F. wird somit von Bruttoinvestitionen ausgegangen, d.h. Abschreibungen, Privatisierungserlöse, Darlehensrückzahlungen sowie Zuschüsse von anderen Ebenen, die dort bereits als Investitionen gebucht wurden, werden nicht herausgerechnet bzw. als investitionsmindernd berücksichtigt. Der Sachverständigenrat zur Begutachtung der gesamtwirtschaftlichen Entwicklung plädiert daher für die Verwendung der um Abschreibungen und Privatisierungserlöse korrigierten Nettoinvestitionen. Bund und einige Bundesländer verwenden jedoch bereits nur die eigenfinanzierten Investitionen zur Berechnung der Kreditobergrenze. Daher erscheint es sachlogisch, in Anlehnung an die sog. „Goldene Regel“ der Haushaltsplanung des Art. 115 GG a.F. die Investitionsausgaben im Haushaltsvollzug der Nettokreditaufnahme - Schuldenaufnahme abzüglich Schuldentilgung - gegenüberzustellen. Dies bedeutet allerdings nicht, dass es sich um verfassungswidrige Haushalte handelt, welche letztlich nur durch Verfassungsgerichte festgestellt werden können. Zumeist wurden die Überschreitungen durch die salvatorischen Klauseln der Abwehr eines gesamtwirtschaftlichen Ungleichgewichts noch gerechtfertigt. Groneck und Kitterer kommen in ihrer Auswertung für den Zeitraum von 1992 bis 2005 insgesamt 66 Überschreitungen der Kreditobergrenze im Vollzug (vgl. Groneck, M./Kitterer, W.: Dauerhafte Verschuldungsregeln für die Bundesländer, in: Wirtschaftsdienst 9 (2006), 559-563). 
konto erfasst; Belastungen, die den Schwellenwert von 1,5 vom Hundert im Verhältnis zum nominalen Bruttoinlandsprodukt überschreiten, sind konjunkturgerecht zurückzuführen. Näheres, insbesondere die Bereinigung der Einnahmen und Ausgaben um finanzielle Transaktionen und das Verfahren zur Berechnung der Obergrenze der jährlichen Nettokreditaufnahme unter Berücksichtigung der konjunkturellen Entwicklung auf der Grundlage eines Konjunkturbereinigungsverfahrens sowie die Kontrolle und den Ausgleich von Abweichungen der tatsächlichen Kreditaufnahme von der Regelgrenze, regelt ein Bundesgesetz. Im Falle von Naturkatastrophen oder außergewöhnlichen Notsituationen, die sich der Kontrolle des Staates entziehen und die staatliche Finanzlage erheblich beeinträchtigen, können diese Kreditobergrenzen auf Grund eines Beschlusses der Mehrheit der Mitglieder des Bundestages überschritten werden. Der Beschluss ist mit einem Tilgungsplan zu verbinden. Die Rückführung der nach Satz 6 aufgenommenen Kredite hat binnen eines angemessenen Zeitraumes zu erfolgen."

Dass diese neue Regel eine deutliche Verschärfung des Stabiltäts- und Wachstumspaktes darstellt, der ja ein Defizit in Höhe von 3 Prozent des BIP zulässt, fand in der Öffentlichkeit kaum Beachtung. Langfristig wird dadurch auch die Schuldenquote deutlich sinken. Für die Abschätzung eines solchen „Grenzwertes der Verschuldung“ kann auf das so genannte „Domar-Modell“ zurückgegriffen werden. ${ }^{48}$ Eine Simulation auf Basis der dort beschriebenen schuldenarithmetischen Zusammenhänge ermöglicht es, den Einfluss verschiedener makroökonomischer Größen auf den Schuldenstand zu beurteilen. Demnach tendiert die Verschuldungsquote in einem Modell mit Inflation zu einem Grenzwert, der sich als Quote aus dem Anteil der Kreditaufnahme am Volkseinkommen im Verhältnis zur Summe aus dem Wirtschaftswachstum und der Inflation berechnet. Bei einem zulässigen Defizit von 0,35 Prozent des BIP, einer Inflationsrate von 2 Prozent und einem Wirtschaftswachstum von 1,5 Prozent würde die Schuldenquote Deutschlands damit auf lange Sicht von aktuell über 82 Prozent auf 23 Prozent sinken, unter Berücksichtigung eines verpflichtenden Haushaltsausgleichs der Bundesländer.

48 Domar, E. D.: The "Burden of the Debt" and the National Income, in: The American Economic Review, 34/4, (1944), 798-827; Blaas, W. /Matzner, E.,: Nachlassendes wirtschaftliches Wachstum und Staatsverschuldung, in: Simmert, D. B./Blaas, W. (Hg.): Staatsverschuldung kontrovers. Köln, 1981, 118-136. 


\section{Der kantonale Vergleich für die Schweiz}

Eine der wohl innovativsten Regeln zur Begrenzung der Staatsverschuldung ist die Schweizer Schuldenbremse, die 2001 für den Bund in einer Volksabstimmung von über 84 Prozent der Schweizer Stimmbürger angenommen wurde. Ursache ihrer Einführung war ein starker, innerhalb der OECD-Länder weit überdurchschnittlicher Verschuldungsanstieg seit Anfang der 1990er Jahre. Bemerkenswert an ihr ist nicht nur der Zwang zum Ausgleich der Staatsverschuldung über einen Konjunkturzyklus hinweg, sondern die gleichzeitige Operationalisierung des „Wie“.

In ihrer Wirkungsweise ist die Schuldenbremse als Ausgabenregel angelegt, d.h. die Ausgaben dürfen nur so hoch ausfallen, wie die mit einem Konjunkturfaktor verrechneten Einnahmen. Läuft die Konjunktur schlecht, dann ist eine stärkere Verschuldung erlaubt. Umgekehrt müssen Überschüsse erzielt werden, sobald die Konjunktur anzieht. Diese werden dann auf einem Ausgleichskonto gutgeschrieben, das wiederum durch Defizite belastet wird und über einen Konjunkturzyklus ausgeglichen sein muss. Dieser Mechanismus führt dazu, dass die Schuldenquote bei wachsender Wirtschaft reduziert wird, was in den vergangenen Jahren auch empirisch zu beobachten war.

Insgesamt kann der Schweizer Schuldenbremse ein großer Erfolg zugesprochen werden. Kritiker dieser Vorlage, wie etwa der Ökonom Beat Kappeler, halten diese neu eingefügte Verfassungsvorschrift jedoch für eine „Mogelpackung“, da Art. 126 (1) der Bundesverfassung von 1999 bereits einen Haushaltsausgleich vorsah und Art. 126 Abs. 2 a.F. überdies einen Abbau der Verschuldung vorschrieb. Durch die Verfassungsänderung wurde dieser zweite Absatz abgeschafft und durch eine deutlich weichere Vorschrift ersetzt, die, so Kappeler, bis Ende $2007 \mathrm{zu}$ einer nominalen Schuldenerhöhung von über 20 Milliarden Franken führte. ${ }^{49}$ Laut Daten des Bundesamtes für Statistik reduzierte sich jedoch von 2003 bis 2009 die nominale Verschuldung auf allen staatlichen Ebenen. Auch die Verwaltung sieht in der neuen Schulenbremse einen substanziellen Fortschritt gegenüber der alten Regelung. ${ }^{50}$ Dies deckt sich auch mit dem bereits benannten Konsolidierungsindikator, der die Schweiz (zusammen mit Norwegen) als das erfolgreichste Konsolidierungsland der vergangenen vier Jahre ausweist. Zudem

49 Kappeler, B.: Eine Schuldenbremse, die nicht zieht, NZZ vom 9. Dezember 2007

50 Siegenthaler, P./Zurbrügg, F.: Die Schweizer Schuldenbremse, in: Kastorp, C./Meister-Scheufelen, G./Sundhof, M. (Hg.): Die neuen Schuldenregeln im Grundgesetz. Zur Fortentwicklung der bundesstaatlichen Finanzbeziehungen, Berlin, 2010, 355-368. 
hat sich seit der Einführung der Schuldenbremse die Konsolidierungsperformanz der Schweiz signifikant verbessert, was die Schweizer Regelung zu einem Vorreiter bei der Schuldenbekämpfung macht.

Gesetzliche Regelungen, die die Schuldenaufnahme in einer bestimmten Art und Weise begrenzen bzw. reglementieren, finden sich in 15 der 26 Schweizer Kantone. Dabei besteht eine große Varianz in der Ausgestaltung der Verschuldungsregeln. Im Kanton Appenzell Ausserrhoden (AR) gibt es beispielsweise die Vorgabe eines mittelfristig ausgeglichenen Haushalts. Das Haushaltsdefizit darf indes nicht größer sein als fünf Prozent der für das laufende Jahr budgetierten Staats- oder Gemeindesteuern, Defizite sind in einem Zeitraum von sieben Jahren abzutragen. Dagegen verzichtet der Kanton Basel-Land in der Finanzhaushaltsordnung jenseits der Forderung der Verfassung nach einem mittelfristig ausgeglichen Haushalt auf eine explizite Ausgestaltung der Schuldenbremse und etwaiger Tilgungsfristen. Im Kanton Genf wiederum macht Art. 7 des Finanzhaushaltsgesetzes (Loi sur la gestion administrative et financière de L'Etat de Genève, in Kraft seit 2006) die Vorgabe, dass der Haushalt ausgeglichen sein muss. Ein Defizit bzw. Ausgabenüberschuss ist hier auf die Summe der Ausgaben für die Schuldentilgung beschränkt, der Ausgleich des Defizits muss innerhalb von vier Jahren erfolgen. Im Jahr 2006 wurde zudem in einer Volksabstimmung Art. 53 der Kantonsverfassung neu gefasst, der diese Defizitbremse dahingehend verschärft, dass ,,jede Gesetzesänderung zur Sanierung der Kantonsfinanzen dem obligatorischen Referendum unterliegt“. Die Stimmberechtigten müssen sich dabei entweder für eine Reduktion der Ausgaben oder eine Erhöhung der Steuern entscheiden - beide Konsolidierungspfade stehen zur Auswahl. ${ }^{51}$ Vor diesem Hintergrund ist es ein bemerkenswerter und äußerst interessanter empirischer Befund, dass gerade der Kanton Genf trotz seiner strikten Verschuldungsregeln am höchsten verschuldet ist und zudem eine hohe Dynamik beim Schuldenzuwachs aufweist.

Zwar weist die multivariate Analyse von Feld und Kirchgässner zum Einflusses der Verschuldungsregeln auf die Entwicklung der kantonalen Defizite und der kantonalen Verschuldungsstände auf einen eindeutig dämpfenden Effekt von Verschuldungsregeln hin, ${ }^{52}$ doch zeigen die Autoren gleichzeitig, dass sich eine

51 Glaeser, A.: Begrenzung der Staatsverschuldung durch die Verfassung. Ein Vergleich deutscher und schweizerischer Regelungen. In: Die öffentliche Verwaltung, 60/3 (2007), 98-106, hier 103.

52 Feld, L./Kirchgässner, G.: On the Effectiveness of Debt Brakes: The Swiss Experience, in: Neck, R/Sturm, E. (eds.): Sustainability of Public Debt, Cambridge, Mass., 2008, 223-255. 
ganze Reihe anderer Faktoren sehr viel stärker auf die Verschuldung auswirkt als die Existenz von Verfassungsregeln. Hierzu zählen zum Beispiel die Direktdemokratie, der Finanzausgleich (Zuweisungen), der Steuerwettbewerb sowie der kulturelle Faktor der Sprachenzugehörigkeit eines Kantons.

\section{Alternativen zu Verschuldungsverboten}

Die vorangegangene Analyse verdeutlicht, dass die Existenz staatlicher Schuldenbremsen nicht wirklich dazu beiträgt, die steigende Verschuldung in der OECD zu begrenzen. Mit Ausnahme der Schweiz erweisen sich Schuldenregeln in den OECD-Ländern nicht als zentrale Einflussfaktoren für den Erfolg von Konsolidierungsbemühungen. Der internationale Vergleich zeigt sogar, dass zahlreiche erfolgreiche Konsolidierungen nicht aufgrund der Existenz von Schuldenregeln durchgeführt wurden. ${ }^{53}$ Anlass waren vielmehr häufig ein zu hohes Verschuldungsniveau und der Verlust an politischem Handlungsspielraum. Denn, wie es etwa Göran Persson als schwedischer Ministerpräsident einmal ausdrückte: „Wer verschuldet ist, ist nicht frei“ .

Eine Alternative $\mathrm{zu}$ den gebräuchlichen Schuldenregeln ist der sogenannte Commitment-Ansatz, der ein glaubhaftes Bekenntnis politischer Entscheidungsträger zum Schuldenabbau beinhaltet. Dessen Kernpunkte sind die Verstärkung einer mehrjährigen Finanzplanung und die verbindliche Festlegung von Ausgabenniveaus bzw. fixen Budgetzielen zu Beginn einer Legislaturperiode, etwa in Koalitionsverträgen. Damit wird die Haushaltskonsolidierung im Rahmen der Einigung auf ein Regierungs- bzw. Koalitionsprogramms zu einem wichtigen politischen Ziel. Diese weiche Form einer Budgetregel ist gleichzeitig flexibler und praktikabler als die Verankerung entsprechender Normen in der Verfassung. Eine solche Strategie wurde u.a. in den Niederlanden gewählt. Neben der öffentlichen Ankündigung von Ausgaben- bzw. Einnahmezielen ist dabei vor allem die Festschreibung dieser Ziele im Koalitionsvertrag entscheidend - was sich als ein Mechanismus für eine stärkere (politische) Haftung der Entscheidungsträger interpretieren ließe. Allerdings wird gerade diese Form institutioneller Bindung im Fiscal Rule Index der EU am schwächsten bewertet.

53 Dieser empirische Befund wird auch durch andere Autoren gestützt. Vgl. z.B. Dreyer Lassen, D.: Fiscal Consolidations in Advanced Industrialized Democracies: Economics, Politics, and Governance, Rapport till Finanspolitiska rådet 2010/4, Stockholm, 2010. 
Eine andere Alternative wird unter dem Schlagwort „Delegation-Ansatz“ diskutiert. ${ }^{54}$ Dieser hat zum Inhalt, die Position des Finanzministers mit sehr viel mehr Macht und Kompetenzen auszustatten. Dessen ureigenes Interesse liege in gesunden Finanzen, und zur Erreichung dieses Ziels müsse er sich in den Budgetberatungen den Wünschen der Fachminister erfolgreich widersetzen können. Der „Delegation-Ansatz“ wird wie der „Commitment-Ansatz“ vom internationalen Vergleich gestützt. So fallen die Staatsschulden geringer aus und die Konsolidierung kommt besser voran, wenn der Finanzminister ein Vetorecht bei der Entscheidung über Ausgaben besitzt - wobei dieses Veto erst durch das funktionierende Zusammenspiel zwischen Premierminister und Finanzminister seine volle Wirkung entfaltet.

In vielen Ländern, die erfolgreiche Konsolidierungsphasen aufzuweisen haben, ${ }^{55}$ etwa in Schweden und den Niederlanden, aber auch in der Schweiz, wurde zudem der Budgetprozess vereinfacht und in einen Top-Down-Ansatz überführt. Auch die Bundesregierung hat in diesem Jahr das traditionelle Anmeldeverfahren im Budetprozess (Bottom-Up-Ansatz) auf ein Top-Down-Verfahren umgestellt: „Das regierungsinterne Verfahren zur Aufstellung des Bundeshaushalts 2012 und des Finanzplans des Bundes 2011 bis 2015 erfolgt im Rahmen eines Top-Down-Verfahrens. Hierzu wird das Bundeskabinett spätestens Mitte März 2011 auf Vorschlag des Bundesministeriums der Finanzen Eckwerte beschließen, die die Einhaltung der verfassungsrechtlichen Schuldenregel sicherstellen und die verbindliche Grundlage für die weitere Haushaltsaufstellung in den Einzelplänen sind.“ 56

Weitere positive Effekte für die Haushaltskonsolidierung sind bei klaren Vorgaben zum Umgang mit unerwarteten Haushaltsüberschüssen zu erkennen. Diese windfall profits entfalten ihre gewünschte Sparwirkung, wenn sie als außergewöhnliche Einnahme zur Schuldentilgung eingesetzt werden. Dasselbe trifft auf zusätzliche Sondereinnahmen (Versteigerungen von Lizenzen, Goldverkäufe, Privatisierungserlöse u.a.m.) zu. Werden diese ausschließlich in den Schuldenabbau gelenkt, wirkt dies über den sinkenden Schuldendienst positiv auf den Finanzierungssaldo zurück.

54 Hallerberg, M./Strauch, R./Hagen, J.v.: Fiscal Governance in Europe, Cambridge, 2009.

55 Wagschal, U./Wenzelburger, G.: Haushaltskonsolidierung, Wiesbaden, 2008.

56 Bundesministerium der Finanzen: Eckwertebeschluss zum Regierungsentwurf des Bundeshaushalts und zum Finanzplan 2011-2015, Berlin, 2011. 
Schließlich zeigt ein näherer Blick auf einige erfolgreiche Konsolidierungsfälle, dass auch moderate Steuererhöhungen, insbesondere für Besserverdienende, eine gewisse Rolle bei der Gesundung der Staatsfinanzen spielen. Gleichwohl sind es vor allem Ausgabenkürzungen, die für eine erfolgreiche Haushaltskonsolidierung verantwortlich sind. Sowohl der bundesstaatliche als auch der internationale Vergleich zeigen: Eine nachhaltige Konsolidierung ist erst durch substanzielle Ausgabenkürzungen möglich.

\section{Fazit}

Der wissenschaftliche und der politische mainstream sehen Schulden- und Haushaltsausgleichsregeln als weitgehend positiv. Unter den Ökonomen bleibt das Lob für Schuldenregeln fast unwidersprochen. ${ }^{57}$ Angesichts der empirischen Erfahrungen und der komparativen Evidenz muss allerdings Wasser in den Wein gegossen werden: Schuldenregeln sind weit weniger wirkungsvoll als immer wieder betont wird. Folgende Ergebnisse können festgehalten werden:

- Verschuldungsregeln konnten Finanzkrisen und Höchststände bei der Verschuldung in vielen Ländern nicht verhindern.

- Länder mit Fiskalregeln verzeichnen einen niedrigeren Verschuldungszuwachs als Länder mit keinen bzw. nur schwachen Fiskalregeln.

- Fiskalregeln zeitigen nahezu keinen Einfluss auf die Haushaltskonsolidierung, also die Rückführung der Staatsverschuldung. Hier sind andere Faktoren entscheidender, etwa das politische Bekenntnis zum Abbau der Verschuldung.

Daher muss die Ausgangsfragestellung dahingehend beantwortet werden, dass Budgetregeln oftmals nicht mehr als Mimikry sind, die einen „gesicherten“ Haushalt vortäuschen. Im Wesentlichen dienen diese Regeln zur Beruhigung der Wähler und der Finanzmärkte, aber auch als strategisches Instrument, um anstehende Konsolidierungen zu begründen. Auch wird deutlich: Haushalte mit Schulden- bzw. Budgetregeln erweisen sich zwar als gesünder als solche ohne Budgetregeln, können eine - im Zweifel zu hohe - Verschuldung allerdings kaum verhindern.

Überdies zeigt der Vergleich, dass zahlreiche Probleme für eine bessere Regelbefolgung ungelöst bleiben. Hierzu zählt vor allem die Durchsetzung der Regeln. Dass mehr und härtere Sanktionen die Lösung sind, darf bezweifelt werden. Hoch defizitäre Länder im Falle einer drohenden Insolvenz mit Sanktionszah- 
lungen zu belegen, scheint vor dem Hintergrund von Konsolidierungserfordernissen kontraproduktiv. Bessere Frühwarnsysteme sind sicher praktikabler, aber auch hier zeigt sich, dass man sich bei den Kriterien für den deutschen Stabilitätsrat eben nur auf den kleinsten gemeinsamen Nenner einigen konnte.

Zur Lösung des Überwachungsproblems hat der Präsident des Kieler Weltwirtschaftsinstituts Snower jüngst eine unabhängige Kommission vorgeschlagen, die in jedem Land die bindenden Etat-Vorgaben der Regierung überwachen soll. Ähnliches hatte bereits 2005 der Genfer Ökonom Wyplosz angeregt, indem er die Errichtung von „finanzpolitischen Komitees“ befürwortete, deren Mitglieder, ähnlich wie die Direktoren einer Zentralbank, unabhängige und nicht vom Wahlvolk zu benennende Experten sein sollen. Die Komitees sollten dann über die Höhe des Haushaltsdefizits entscheiden, nicht aber über die Zusammensetzung der Ausgaben. Seine Diktum: Institutionen sind besser als Regeln. ${ }^{58}$

Ein Grundproblem von Budgetregeln liegt zudem in der ihnen mangelnden Flexibilität; im Zweifel könnte der Staat nicht mehr auf Krisen oder auf Konjunktureinbrüche reagieren. Hier lässt der neue Art. 115 GG zwar Spielraum (wie im Übrigen auch Art. 115 GG a.F.), unklar bleibt jedoch die Definition solcher Ausnahmetatbestände und Krisen. Die Politik hat darüber hinaus auch ein Glaubwürdigkeitsproblem bei der Durchsetzung von Regeln: Konrad bezeichnet dies, allerdings empirisch unbelegt, als Samariterdilemma, da die Politik aus Solidaritätsgründen in Mehrebenensystemen moralisch zur Hilfe verpflichtet sei. ${ }^{59}$

Die Regelbefolgung variiert zwischen den EU-Mitgliedstaaten schon seit langem, etwa bei der Umsetzung europäischer Richtlinien. Dies hat auch mit der Akzeptanz der Vorschriften zu tun. Werden Haushaltsregeln als unverbindliche Kenngrößen gesehen, die traditionell im politischen System eines Landes nicht vorhanden waren, kann eine dauerhafte compliance kaum erwartet werden. Ungelöst bleibt bei aller Regelbindung und Begrenzung der Verschuldung die Frage, wie Staaten eine Haushaltskonsolidierung bewerkstelligen sollen, wenn keine Änderung der Ausgaben- und Aufgabenstruktur zu erwarten ist. Gesetzliche Ansprüche und die ausgeprägte Belastung des Haushalts durch ex ante festgelegte Positionen lassen sich nur durch politische Reformen ändern, also durch diskretionäre Politik.

58 Snower, D.: Einfache Lösung für Euro-Krise, in: Frankfurter Rundschau vom 26.08.2011; Wyplosz, C.: Fiscal Policy: Institutions versus Rules, in: National Institute Economic Review 191 (2005), 64-78-

59 Konrad, K.: Fiskalregeln und Zeitinkonsistenz, in: Kastrop, C./Meister-Scheufelen, G./Sudhof, M. (Hrsg.): Die neuen Schuldenregeln im Grundgesetz, Berlin, 2010. 